\title{
EXISTENCE AND DUALITY THEOREMS FOR CONVEX PROBLEMS OF BOLZA
}

\author{
BY \\ R. T. ROCKAFELLAR( $\left.{ }^{1}\right)$
}

\begin{abstract}
The theory of conjugate convex functions is applied to a fundamental class of "convex" problems in the calculus of variations and optimal control. This class has many special properties which have not previously been exploited and for which the standard methods of approach are inadequate. Duality theorems are established which yield new results on the existence of optimal arcs, as well as necessary and sufficient conditions for optimality. These results have some relevance also to the study of "nonconvex" problems.
\end{abstract}

Introduction. Many problems in the calculus of variations and optimal control can be formulated as generalized problems of Bolza. We have shown in [9] that if certain convexity assumptions and mild regularity assumptions (not requiring differentiability) are satisfied, such a problem has associated with it a dual problem, which is likewise a generalized problem of Bolza. The dual of the dual problem is the original problem.

The main result of this paper, Theorem 1 (formulated in $\S 1$ ), relates the extremal values in a dual pair of problems of Bolza and gives conditions under which optimal arcs exist. This theorem also provides a condition for the weak compactness of certain subsets of the (nonreflexive) Banach space of all absolutely continuous arcs $x:[0, T] \rightarrow R^{n}$. It establishes (Corollary 1) a class of problems for which the generalized Euler-Lagrange equation (or Hamiltonian equation) and transversality condition studied in [9] and [10] are necessary and sufficient if an arc is to be optimal.

Duality in various forms has already been investigated in optimal control and the calculus of variations by a number of authors (see the remarks and references in [9]), but the duality content of Theorem 1 is not immediately comparable with anything in this literature. The pattern is that of the general duality theorems that have been developed for convex programs, and the result itself sharpens Theorem 3 in our preceding paper [9]. However, there is an essential difference. The previous theorems applicable to convex problems of Bolza have hypotheses concerning a convex subset of the dual of a nonreflexive Banach space: in effect, certain points are

Received by the editors August 31, 1970.

AMS 1970 subject classifications. Primary 49A10, 49B10; Secondary 46E15.

Key words and phrases. Optimal control, problem of Bolza, dual minimization problems, convex Lagrangian functions, Hamiltonian functions, existence of solutions, necessary conditions, conjugate convex functions.

(1) This work was supported in part by grant AFOSR-71-1994.

Copyright (C) 1971, American Mathematical Society 
assumed to belong to the weak* closure or Mackey interior of this set. Needless to say, such conditions can be very hard to verify. The chief contribution of Theorem 1 is to furnish, despite the underlying nonreflexivity, alternative "finitedimensional" conditions stated directly in terms of the given Lagrangian functions, Hamiltonian functions, boundary functions, and their growth properties. Most of the other theorems and propositions in this paper are aimed at the elucidation of these conditions and their consequences.

A precise comparison of the existence content of Theorem 1 with other results on the existence of optimal arcs is difficult. Different authors adopt different basic models, and much reformulation is necessary in passing from one context to another. Among recent papers on the subject, those of Cesari [2] and Olech [5] on problems of Lagrange are the most relevant to the present work. However, the problems treated in these papers are subjected to stronger assumptions of continuity and boundedness, yet less restrictive assumptions of convexity, than the ones treated here. For the class of problems to which all the theories are immediately applicable, it appears that our existence results are sharper, although not greatly different in scope.

The principal distinction is that, in the theorems of Cesari and Olech, boundedness conditions (needed in compactness arguments) are introduced through the specification of a class of "admissible" arcs over which the given functional is to be minimized. Such conditions do not appear explicitly in Theorem 1. Instead, boundedness conditions, to the extent that they are present at all, are incorporated into the growth conditions on the given functions.

In fact, rather than assuming much boundedness, Theorem 1 in its compactness assertion provides a new criterion for boundedness. This criterion is applicable even to problems not satisfying our convexity assumptions, and thus it could lead to further extensions of existence theory. We do not pursue this below, but the idea is quite simple: If a general Bolza functional (not necessarily convex) majorizes a convex Bolza functional satisfying all the conditions in Theorem 1(a), then its level sets are relatively compact in the weak topology, and in particular bounded.

The approach we use to derive the existence of optimal arcs is entirely different from the usual approach, where it is shown that a minimizing sequence of arcs has a subsequence converging in some sense to a solution to the problem. We get existence essentially by invoking a separation theorem. A major complication is the fact that the separation theorem is invoked in the dual of a nonreflexive Banach space. It must be shown by a lengthy argument that the separating hyperplane can actually be represented by an element of the original space, rather than the bidual space. Of course, this approach to existence theory is not possible for problems not satisfying our convexity assumptions, although, as mentioned above, results in the convex case can be applied to more general cases indirectly.

The necessary conditions for optimality that we obtain from Theorem 1 have already been analyzed in detail in [9] and [10], and we do not develop them further 
here. These conditions, involving subgradients of convex functions, are known always to be sufficient [9, Theorem 5], a fact which serves to emphasize the special nature of convex problems of Bolza and the desirability of a separate treatment of such problems. Particularly in computational work, it is valuable to have conditions for optimality that are both necessary and sufficient. Thus, hopefully, the results in this paper could lead to improved algorithms. The necessary and sufficient conditions, as well as the results on existence and duality, may also be helpful in applications of optimal control to areas like economic growth theory, where convexity is often very appropriate but the differentiability assumptions typical of many physical applications are rather unnatural.

Our conditions for optimality depend on strong convexity assumptions, but again this does not necessarily mean that they do not have a bearing on "nonconvex" problems. After all, much of variational theory involves notions of local linearization or convexification. One may speculate that a well-developed theory of "convexified problems" could lead to further progress along such lines. This, at least, is one of the motivations for studying convex problems of Bolza.

The detailed plan of this paper is described at the end of $\S 1$, after the exact statement of the main theorem and its corollaries.

1. Statement of the main theorem. To reduce the length of the exposition, we assume familiarity with the concepts and definitions in our previous paper on dual problems of Bolza [9]. However, we repeat for easy reference the description of the problem and the fundamental assumptions.

Let $[0, T]$ be a fixed real interval $(0<T<+\infty)$, and let $L_{n}^{p}$ denote the usual Banach space of (equivalence classes of) summable functions from $[0, T]$ to $R^{n}$ (the latter under the Euclidean norm $|\cdot|)$. The norm on $L_{n}^{p}$ is denoted by $\|\cdot\|_{p}$. Let $A_{n}^{1}$ be the Banach space consisting of all absolutely continuous arcs $x:[0, T] \rightarrow R^{n}$ under the norm

$$
\|x\|=|x(0)|+\int_{0}^{T}|\dot{x}(t)| d t .
$$

We consider the problem of minimizing over $A_{n}^{1}$ a functional of the form

$$
\Phi_{l, L}(x)=l(x(0), x(T))+\int_{0}^{T} L(t, x(t), \dot{x}(t)) d t,
$$

where $l$ and $L_{t}=L(t, \cdot, \cdot)$ are (everywhere defined) functions on $R^{n} \times R^{n}$ with values in $R^{1} \cup\{+\infty\}$. This is called a convex problem of Bolza if the following conditions are satisfied, as we henceforth always assume.

(A) Each of the functions $l$ and $L(t, \cdot, \cdot)$ is convex, lower semicontinuous, and not identically $+\infty$.

(B) $L$ is measurable with respect to the $\sigma$-field in $[0, T] \times R^{n} \times R^{n}$ generated by products of Lebesgue sets in [0,T] and Borel sets in $R^{n} \times R^{n}$, or equivalently [8], $L$ is a normal convex integrand in the sense of [7]. 
(C) There exist functions $p \in L_{n}^{\infty}, s \in L_{n}^{1}$ and $\alpha \in L_{1}^{1}$ such that

$$
L(t, x, v) \geqq\langle x, s(t)\rangle+\langle v, p(t)\rangle-\alpha(t) .
$$

(D) There exist functions $x \in L_{n}^{\infty}, v \in L_{n}^{1}$ and $\beta \in L_{1}^{1}$ such that $L(t, x(t), v(t)) \leqq \beta(t)$. These conditions are discussed in [9], where various examples of convex problems of Bolza are given. They imply in particular that, for every $x \in L_{n}^{\infty}$ and $v \in L_{n}^{1}$, $L(t, x(t), v(t))$ is a measurable function of $t$ which majorizes at least one summable function of $t$. In fact, $\Phi_{l, L}$ is a well-defined functional from $A_{n}^{1}$ to $R^{1} \cup\{+\infty\}$ which is convex and (weakly and strongly) lower semicontinuous [9, Theorem 1]. Conditions (B), (C), and (D) are trivially satisfied if $L$ is independent of $t$.

Every $\operatorname{arc} x \in A_{n}^{1}$ such that $\Phi_{l, L}(x) \neq+\infty$ satisfies

$$
(x(0), x(t)) \in C_{l},
$$

$$
(x(t), \dot{x}(t)) \in D_{L}(t) \text { for almost every } t,
$$

where $C_{l}$ and $D_{L}(t)$ are the (nonempty, convex) effective domains of $l$ and $L_{t}$ :

$$
\begin{gathered}
C_{l}=\left\{\left(c_{0}, c_{T}\right) \in R^{n} \times R^{n} \mid l\left(c_{0}, c_{T}\right)<+\infty\right\}, \\
D_{L}(t)=\left\{(x, v) \in R^{n} \times R^{n} \mid L(t, x, v)<+\infty\right\} .
\end{gathered}
$$

Thus minimizing $\Phi_{l, L}$ over $A_{n}^{1}$ is equivalent to minimizing $\Phi_{l, L}$ subject to (1.2) and (1.3).

The dual problem of Bolza consists of minimizing

$$
\Phi_{m, M}(p)=m(p(0), p(T))+\int_{0}^{T} M(t, p(t), \dot{p}(t)) d t
$$

over $A_{n}^{1}$, where $m$ and $M_{t}=M(t, \cdot, \cdot)$ are defined by

$$
\begin{gathered}
m\left(d_{0}, d_{T}\right)=l^{*}\left(d_{0},-d_{T}\right)=\sup _{c_{0}, c_{T}}\left\{\left\langle c_{0}, d_{0}\right\rangle-\left\langle c_{T}, d_{T}\right\rangle-l\left(c_{0}, c_{T}\right)\right\}, \\
M_{t}(p, s)=L_{t}^{*}(s, p)=\sup _{x, v}\{\langle x, s\rangle+\langle v, p\rangle-L(t, x, v)\} .
\end{gathered}
$$

(Here $\langle\cdot, \cdot\rangle$ denotes the inner product in $R^{n}$, and the suprema are taken over all of $R^{n} \times R^{n}$.) An asterisk marks the conjugate of a convex function. The dual functions $m$ and $M$ again satisfy (A), (B), (C), and (D), and their duals are in turn $l$ and $L$ [9, Theorem 2]. Conditions (C) and (D) are dual to each other, in the sense that functions $s, p, \alpha$ have the property in (C) if and only if $M(t, p(t), s(t)) \leqq \alpha(t)$, whereas functions $x, v$ and $\beta$ have the property in (D) if and only if

$$
M(t, p, s) \geqq\langle p, v(t)\rangle+\langle s, x(t)\rangle-\alpha(t) .
$$

Minimizing $\Phi_{m, M}$ over all of $A_{n}^{1}$ is equivalent to minimizing $\Phi_{m, M}$ subject to the constraints

$$
(p(0), p(T)) \in C_{m},
$$

$$
(p(t), \dot{p}(t)) \in D_{M}(t) \text { for almost every } t \text {, }
$$


where

$$
\begin{gathered}
C_{m}=\left\{\left(d_{0}, d_{T}\right) \in R^{n} \times R^{n} \mid m\left(d_{0}, d_{T}\right)<+\infty\right\}, \\
D_{M}(t)=\left\{(p, s) \in R^{n} \times R^{n} \mid M(t, p, s)<+\infty\right\} .
\end{gathered}
$$

(The convex sets $C_{m}$ and $D_{M}(t)$ can be determined from the recession functions of $l$ and $L_{t}$; see $\$ 4$ and [6, Theorem 13.3].)

As shown in [9], one always has the inequality

$$
\inf _{x \in A_{n}^{1}} \Phi_{l, L}(x) \geqq-\inf _{p \in A_{n}^{1}} \Phi_{m, M}(p),
$$

and equality holds if and only if the Bolza functionals $\Phi_{l, L}$ and $\Phi_{m, M}$ " behave lower semicontinuously with respect to certain perturbations." Theorem 1 , the existence and duality result below, gives conditions on $l, L, m$ and $M$ for equality to hold in (1.13) and for the infima to be attained. These conditions are of two types: stronger forms of (C) and (D) and conditions on the attainability of endpoint pairs in the sets $C_{l}$ and $C_{m}$.

The stronger forms of (C) and (D) are

$\left(\mathrm{C}_{0}\right)$ For each $p \in R^{n}$ there exist functions $s \in L_{n}^{1}$ and $\alpha \in L_{1}^{1}$ such that $L(t, x, v)$ $\geqq\langle x, s(t)\rangle+\langle v, p\rangle-\alpha(t)$.

$\left(\mathrm{D}_{0}\right)$ For each $x \in R^{n}$ there exist functions $v \in L_{n}^{1}$ and $\beta \in L_{1}^{1}$ such that $L(t, x, v(t))$ $\leqq \beta(t)$.

Conditions $\left(\mathrm{C}_{0}\right)$ and $\left(\mathrm{D}_{0}\right)$ are dual to each other, like (C) and (D): $L$ has the property in $\left(\mathrm{C}_{0}\right)$ if and only if $M$ has the property in $\left(\mathrm{D}_{0}\right)$, and vice versa. We show in $\$ 2$ that these conditions can be expressed in an equivalent, but seemingly weaker, manner in terms of the Hamiltonian function

$$
H(t, x, p)=\sup \left\{\langle v, p\rangle-L(t, x, v) \mid v \in R^{n}\right\} .
$$

The Hamiltonian form of $\left(\mathrm{C}_{0}\right)$ corresponds to a basic condition employed in existence theory by Olech [5].

Both $\left(\mathrm{C}_{0}\right)$ and $\left(\mathrm{D}_{0}\right)$ hold if and only if $H(t, x, p)$ is finite and summable in $t$ for every $(x, p) \in R^{n} \times R^{n}(\S 2$, Corollary to Proposition 4$)$. In the case where $L$ is independent of $t,\left(\mathrm{D}_{0}\right)$ holds if and only if $H$ nowhere has the value $-\infty$, while $\left(\mathrm{C}_{0}\right)$ holds if and only if $H$ nowhere has the value $+\infty$ (see Proposition 1 in $\S 2$ ). In essence, $\left(\mathrm{C}_{0}\right)$ is a growth condition on the convex functions $L(t, x, \cdot)$ resembling the classical growth conditions of Nagumo and Tonelli (see [2, p. 403] and the references given there).

Observe that $\left(D_{0}\right)$ precludes the presence of "implicit state constraints" in the problem of Bolza for $l$ and $L$, since it implies that for every $t \in[0, T]$ and $x \in R^{n}$ there is at least one admissible choice of $v$, that is, at least one $v \in R^{n}$ such that $L(t, x, v)<+\infty$. Similarly, $\left(\mathrm{C}_{0}\right)$ precludes the presence of "implicit state constraints" in the problem of Bolza for $m$ and $M$. More is said about this below. 
We call an endpoint pair $\left(c_{0}, c_{T}\right) \in R^{n} \times R^{n}$ attainable for $L$ if there is an arc $x \in A_{n}^{1}$ such that

$$
x(0)=c_{0}, \quad x(T)=c_{T} \text { and } \int_{0}^{T} L(t, x(t), \dot{x}(t)) d t<+\infty,
$$

and weakly attainable for $L$ if there is an arc $x \in A_{n}^{1}$ such that

$$
x(0)=c_{0}, \quad x(T)=c_{T} \quad \text { and } \quad(x(t), \dot{x}(t)) \in \mathrm{cl} D_{L}(t) \quad \text { a.e. }
$$

Relationships between these concepts are treated in $\S 4$ (Corollary 4 of Theorem 3), but the weaker concept is the one used in stating Theorem 1 .

The set of all weakly attainable pairs $\left(c_{0}, c_{T}\right)$ for $L$ is denoted by $C_{L}$. Similarly, $C_{M}$ denotes the set of all $\left(d_{0}, d_{T}\right) \in R^{n} \times R^{n}$ for which there exists an arc $p \in A_{n}^{1}$ such that

$$
p(0)=d_{0}, \quad p(T)=d_{T} \quad \text { and } \quad(p(t), \dot{p}(t)) \in \operatorname{cl} D_{M}(t) \quad \text { a.e. }
$$

Obviously $C_{L}$ and $C_{M}$ are convex. Furthermore, if $\Phi_{l, L} \not \equiv+\infty$ we have $C_{l} \cap C_{L} \neq \varnothing$, while if $\Phi_{m, M} \not \equiv+\infty$ we have $C_{m} \cap C_{M} \neq \varnothing$. In Theorem 1 it is required that these intersections remain nonempty if the sets are replaced by their relative interiors.

We denote by aff $C$ the affine hull of a set $C$ (the smallest affine set containing $C$, an affine set being either the empty set or a translate of a subspace). If $C$ is convex, we denote by ri $C$ the relative interior of $C$ (the interior of $C$ relative to aff $C$ ).

THEOREM 1. (a) If condition $\left(\mathrm{C}_{0}\right)$ holds and ri $C_{m} \cap$ ri $C_{M} \neq \varnothing$, then

$$
\min _{x \in A_{n}^{1}} \Phi_{l, L}(x)=-\inf _{p \in A_{n}^{1}} \Phi_{m, M}(p)>-\infty .
$$

If in addition aff $\left(C_{m} \cup C_{M}\right)=R^{n} \times R^{n}$, then the convex level sets

$$
\left\{x \in A_{n}^{1} \mid \Phi_{l, L}(x) \leqq \mu\right\}, \quad \mu \in R^{1},
$$

are weakly compact in $A_{n}^{1}$.

(b) If condition $\left(\mathrm{D}_{0}\right)$ holds and ri $C_{l} \cap$ ri $C_{L} \neq \varnothing$, then

$$
\inf _{x \in A_{n}^{1}} \Phi_{l, L}(x)=-\min _{p \in A_{n}^{1}} \Phi_{m, M}(p)<+\infty .
$$

If in addition aff $\left(C_{l} \cup C_{L}\right)=R^{n} \times R^{n}$, then the convex level sets

$$
\left\{p \in A_{n}^{1} \mid \Phi_{m, M}(p) \leqq \mu\right\}, \quad \mu \in R^{1},
$$

are weakly compact in $A_{n}^{1}$.

Here we use the convention of writing "min" in place of "inf" to indicate that an infimum is attained. Thus (a) asserts in particular that an optimal arc exists for the problem of Bolza for $l$ and $L$, unless there is no feasible arc at all, which corresponds to the case where $\Phi_{m, M}$ is not bounded below. Similarly, (b) asserts that an optimal arc exists for $m$ and $M$, unless there is no feasible arc at all, which corresponds to the case where $\Phi_{l, L}$ is not bounded below. 
Note that parts (a) and (b) of Theorem 1 imply each other by duality. Therefore in developing the proof of Theorem 1 we concentrate on (b) and state many results for this case only, although such results could easily be dualized. However, we treat the assumptions in (a) in considerable detail, since it may be desirable to use (a) to deduce the existence of optimal arcs for $l$ and $L$ without explicitly determining the dual functions $m$ and $M$. To this end, one can apply to $l$ and $L$ other versions of the conditions in (a) which we establish below.

In particular, it is shown in $\S 4$ (Corollary 1 to Theorem 3 ) that, in the case where $\left(\mathrm{C}_{0}\right)$ is satisfied and $\Phi_{l, L}$ is not identically $+\infty$ on $A_{n}^{1}$, one has

$$
\text { ri } C_{m} \cap \text { ri } C_{M} \neq \varnothing \text { and aff }\left(C_{m} \cup C_{M}\right)=R^{n} \times R^{n}
$$

if and only if there is no arc $z \in A_{n}^{1}$, other than $z=0$, such that $\Phi_{l, L}(x+\lambda z)$ is a nonincreasing function of $\lambda \in R^{1}$ for every $x \in A_{n}^{1}$. The latter condition can itself be expressed in terms of the growth properties of the convex functions $l$ and $L_{t}$ themselves (Proposition 6): $\Phi_{l, L}(x+\lambda z)$ is nonincreasing in $\lambda$ for every $x$ if and only if $z$ satisfies

$$
\hat{l}(z(0), z(T))+\int_{0}^{T} \hat{L}(t, z(t), \dot{z}(t)) d t \leqq 0,
$$

where $\hat{l}$ and $\hat{L}_{t}$ are the recession functions [6] of $l$ and $L_{t}$. The condition on $\Phi_{l, L}$ equivalent to (1.22) is obviously satisfied, for example, no matter what the choice of $l$, if there exist real numbers $\rho$ and $\mu$, such that there is at least one arc $x \in A_{n}^{1}$ with $\Phi_{l, L}(x) \leqq \mu$, and every such $x$ has $|x(t)| \leqq \rho, 0 \leqq t \leqq T$. Thus (1.18) holds in particular if $\left(\mathrm{C}_{0}\right)$ holds and there is a unique optimal arc $x$ for $l$ and $L$, or the set of optimal arcs is merely known to be bounded. Example 6 of [9] is also contained as a special case.

Another major class of problems for which the assumptions in Theorem 1(a) are satisfied is described in Corollary 3 of Theorem 3 in $\$ 4$.

Without any condition at all on the sets $C_{m}$ and $C_{M}$, a compactness result generalizing the one in Theorem 1(a) is still obtainable. We prove in $\S 3$ that, whenever $\left(\mathrm{C}_{0}\right)$ holds, the level sets (1.19) of $\Phi_{l, L}$ are locally compact relative to the weak topology on $A_{n}^{1}$ and in particular have the property that every bounded subset is weakly relatively compact. (This is asserted by the dual of Corollary 6 of Theorem 2.)

Although the existence result in (b) concerns the dual problem of Bolza, rather than the original problem, it is also of direct interest, because it yields a necessary and sufficient condition for optimality in the original problem. The following corollary is immediate from Theorem 5 of [9].

Corollary 1. Assume that $\left(\mathrm{D}_{0}\right)$ holds and that ri $C_{l} \cap$ ri $C_{L} \neq \varnothing$. Then, in order that $x \in A_{n}^{1}$ be an arc minimizing $\Phi_{l, L}$, it is necessary and sufficient that $x$ be an extremal arc for $l$ and $L$, or in other words, that $x$ satisfy the generalized EulerLagrange equation (or Hamiltonian equation) and transversality condition in $[9, \S 9]$. 
This fact gives some insight, incidentally, into the role of $\left(D_{0}\right)$ in excluding "implicit state constraints". The dual extremal arc $p$ in the necessary condition referred to in Corollary 1 belongs to $A_{n}^{1}$ (it minimizes $\Phi_{m, M}$ ). However, if state constraints were present, one would expect from well-known results in control theory and the calculus of variations that $p$ would be discontinuous, or at least could not be guaranteed to be absolutely continuous. The methods in this paper could in fact be used to attack this more general situation, but $A_{n}^{1}$ would have to be replaced by a larger space (allowing for "idealized solutions" to a problem of Bolza), and the corresponding duality theory would not be as symmetric. Actually, there is another method whereby necessary and sufficient conditions for optimality in many problems with state constraints can be derived directly from Theorem 1 and general theorems about subgradients. This is treated in [12]. Thus, in the long run, condition $\left(D_{0}\right)$ does not really impose a serious restriction on the applicability of the present theory, but acts more to normalize, for technical convenience, the class of problems under consideration at a particular stage.

Another corollary of Theorem 1 may be obtained by specializing the problems of Bolza to problems of Lagrange. If we take $l$ to be the indicator of a point pair $\left(c_{0}, c_{T}\right) \in R^{n} \times R^{n}$ (that is, the function which vanishes at $\left(c_{0}, c_{T}\right)$ and has the value $+\infty$ everywhere else), then minimizing $\Phi_{l, L}$ over $A_{n}^{1}$ is equivalent to minimizing the integral

$$
\int_{0}^{T} L(t, x(t), \dot{x}(t)) d t
$$

over all arcs $x$ with the fixed endpoints $c_{0}$ and $c_{T}$. In this case, the dual problem consists of minimizing

$$
\left\langle c_{0}, p(0)\right\rangle-\left\langle c_{T}, p(T)\right\rangle+\int_{0}^{T} M(t, p(t), \dot{p}(t)) d t .
$$

Moreover $C_{m}=R^{n} \times R^{n}$ in the dual problem, so that the intersection condition in (a) is satisfied if and only if $C_{M} \neq \varnothing$.

Let $F_{L}$ and $F_{M}$ be the extended-real-valued functions on $R^{n} \times R^{n}$ defined by

$$
\begin{aligned}
& F_{L}\left(c_{0}, c_{T}\right)=\inf \left\{\int_{0}^{T} L(t, x(t), \dot{x}(t)) d t \mid x \in A_{n}^{1}, x(0)=c_{0}, x(T)=c_{T}\right\} \\
& F_{M}\left(d_{0}, d_{T}\right)=\inf \left\{\int_{0}^{T} M(t, p(t), \dot{p}(t)) d t \mid p \in A_{n}^{1}, p(0)=d_{0}, p(T)=d_{T}\right\}
\end{aligned}
$$

It is obvious from the convexity of $L(t, \cdot, \cdot)$ and $M(t, \cdot, \cdot)$ that $F_{L}$ and $F_{M}$ are convex. The preceding observations (and the dual observations) yield modified conjugacy relations between $F_{L}$ and $F_{M}$.

Corollary 2. (a) Suppose that $\left(\mathrm{C}_{0}\right)$ holds, and that $C_{M} \neq \varnothing$. Then the convex function $F_{L}$ is lower semicontinuous and nowhere $-\infty$, and for every choice of endpoints $c_{0}$ and $c_{T}$, the infimum defining $F_{L}\left(c_{0}, c_{T}\right)$ is attained. Moreover, one has

$$
F_{L}\left(c_{0}, c_{T}\right)=F_{M}^{*}\left(-c_{0}, c_{T}\right)=\sup _{d_{0}, d_{T}}\left\{\left\langle c_{T}, d_{T}\right\rangle-\left\langle c_{0}, d_{0}\right\rangle-F_{M}\left(d_{0}, d_{T}\right)\right\}
$$


(b) Suppose that $\left(\mathrm{D}_{0}\right)$ holds, and that $C_{L} \neq \varnothing$. Then the convex function $F_{M}$ is lower semicontinuous and nowhere $-\infty$, and for every choice of endpoints $d_{0}$ and $d_{T}$, the infimum defining $F_{M}\left(d_{0}, d_{T}\right)$ is attained. Moreover, one has

$$
F_{M}\left(d_{0}, d_{T}\right)=F_{L}^{*}\left(-d_{0}, d_{T}\right)=\sup _{c_{0}, c_{T}}\left\{\left\langle c_{T}, d_{T}\right\rangle-\left\langle c_{0}, d_{0}\right\rangle-F_{L}\left(c_{0}, c_{T}\right)\right\} .
$$

The asserted lower semicontinuity properties are immediate from (1.27) and (1.28).

The condition $C_{M} \neq \varnothing$ in part (a) of Corollary 2 is equivalent to the following, unless $C_{L}=\varnothing$, in which event $F_{L}$ would be identically $+\infty$ (see $\S 4$, Corollary 2 to Theorem 3): there is no $\operatorname{arc} z$, other than $z=0$, with the property that $z(0)=0=z(T)$ and the integral

$$
\int_{0}^{T} L(t, x(t)+\lambda z(t), \dot{x}(t)+\lambda \dot{z}(t)) d t
$$

is nonincreasing as a function of $\lambda \in R^{1}$ for every $x \in A_{n}^{1}$. Again, the latter condition can be expressed in terms of recession functions.

Corollary 2 leads to a refinement of Theorem 1 in certain cases where the boundary functions $l$ and $m$ are polyhedral (i.e. have epigraphs which are polyhedral convex sets; see $[6, \S 19])$. This is seen from the fact that, in terms of the function $F_{L}$, the problem of Bolza for $l$ and $L$ can be regarded as the finite-dimensional problem of minimizing

$$
l\left(c_{0}, c_{T}\right)+F_{L}\left(c_{0}, c_{T}\right)
$$

over $R^{n} \times R^{n}$. Fenchel's Duality Theorem [6, Theorem 31.1] can be applied to this type of problem, the dual problem being equivalent, as one would expect, to the problem of minimizing

$$
m\left(d_{0}, d_{T}\right)+F_{M}\left(d_{0}, d_{T}\right)
$$

over $R^{n} \times R^{n}$ if (1.28) holds. From this theorem, one obtains the fact

$$
\inf \left(l+F_{L}\right)=-\min \left(m+F_{M}\right),
$$

if $l$ is polyhedral and $C_{l}$ meets the relative interior of

$$
\left\{\left(c_{0}, c_{T}\right) \mid F_{L}\left(c_{0}, c_{T}\right)<+\infty\right\} .
$$

It is proved below in $\$ 4$ (Corollary 4 of Theorem 3 ), however, that the convex set (1.33), which consists precisely of the attainable endpoint pairs for $L$, has the same relative interior as $C_{L}$. Thus:

Corollary 3. (a) If $\left(\mathrm{C}_{0}\right)$ holds, $l$ is polyhedral, and $C_{m} \cap$ ri $C_{M} \neq \varnothing$, then

$$
\min _{x \in A_{n}^{1}} \Phi_{l, L}(x)=-\inf _{p \in A_{n}^{1}} \Phi_{m, M}(p) .
$$

(b) If $\left(\mathrm{D}_{0}\right)$ holds, $l$ is polyhedral, and $C_{l} \cap$ ri $C_{L} \neq \varnothing$, then

$$
\inf _{x \in A_{n}^{1}} \Phi_{l, L}(x)=-\min _{p \in A_{n}^{1}} \Phi_{m, M}(p) .
$$


Note that here there is no compactness assertion of the kind in Theorem 1. Of course, Corollary 1 is still valid under the hypothesis of Corollary 3(b), when $l$ is polyhedral, since it follows directly from equation (1.35) and Theorem 5 of [9]. Applied to problems of Lagrange, Corollary 1 asserts that, under the hypothesis of Corollary 2(b), the arcs $x$ for which the infima in the definition of $F_{L}$ are attained are precisely the extremals of $L$ (that is, the arcs which satisfy the generalized EulerLagrange equation for $L$; see $[9, \S 9])$.

The plan of the rest of the paper is as follows. $\$ 2$ is of a preliminary nature; it is devoted to a discussion of equivalent and stronger forms of conditions $\left(\mathrm{C}_{0}\right)$ and $\left(D_{0}\right)$. The proof of Theorem 1 really begins in $\S 3$ with the establishment of Theorem 2 and its corollaries, concerning "perturbations" of the two problems of Bolza. These results are the crucial consequences of conditions $\left(C_{0}\right)$ and $\left(D_{0}\right)$. They include (embodied in Corollaries 5 and 6) an existence and duality theorem with the same conclusions as Theorem 1, but with hypotheses that are less direct. Theorem 3 in $\$ 4$ translates these hypotheses into the ones in Theorem 1 concerning the convex sets $C_{l}, C_{L}, C_{m}$ and $C_{M}$. The proof of Theorem 1 is thereby effected.

The last two sections deal with supplementary results. The meaning in a convex problem of Bolza of the optimal arcs for the dual problem is described in $\$ 5$ in terms of the behavior of the primal problem under "perturbations." In $\S 6$, some conclusions are drawn about problems of Bolza in which one minimizes, not over $A_{n}^{1}$, but over $A_{n}^{r}$ (the space of absolutely continuous arcs $x:[0, T] \rightarrow R^{n}$ with derivative $\dot{x}$ in $\left.L_{n}^{r}, 1<r \leqq \infty\right)$. In particular, growth conditions are given on $L(t, x, v)$ which imply that the optimal arcs in the problem of minimizing $\Phi_{l, L}$ over $A_{n}^{1}$ actually belong to $A_{n}^{r}$ for a specified $r>1$.

2. Hamiltonian functions and conditions $\left(C_{0}\right)$ and $\left(D_{0}\right)$. We now treat conditions $\left(C_{0}\right)$ and $\left(D_{0}\right)$ in greater detail, with the aim of deriving equivalent or stronger versions of these conditions which may be easier to verify in some cases.

It is convenient to work with the Hamiltonian function $H$ on $[0, T] \times R^{n} \times R^{n}$ given by (1.14). Formula (1.14) says that $H(t, x, \cdot)$ is conjugate to $L(t, x, \cdot)$, and therefore $L(t, x, \cdot)$ is in turn conjugate to $H(t, x, \cdot)$ :

$$
L(t, x, v)=\sup \left\{\langle v, p\rangle-H(t, x, p) \mid p \in R^{n}\right\} .
$$

It is known that $H(t, x, p)$ is convex in $p$, concave in $x$ and measurable in $t$. In fact, $H(t, x(t), p(t))$ is measurable in $t$ whenever $x(t)$ and $p(t)$ are measurable in $t$ [10, Proposition 1].

From the definitions of $M$ and $H$, we have

$$
M(t, p, s)=\sup \left\{\langle x, s\rangle+H(t, x, p) \mid x \in R^{n}\right\} .
$$

Thus $M(t, p, \cdot)$ is the conjugate of the convex function $-H(t, \cdot, p)$, so that the conjugate of $M(t, p, \cdot)$ is the so-called closure of $-H(t, \cdot, p)$ as a function of $x[6, \S 7$ and $\S 12]$. Therefore, if we denote by $\tilde{H}$ the Hamiltonian function which 
corresponds to $M$ in the same way that $H$ corresponds to $L$, we have

$$
\begin{gathered}
\tilde{H}(t, p, x)=-\operatorname{cl}_{x} H(t, x, p), \\
H(t, x, p)=-\operatorname{cl}_{p} \tilde{H}(t, p, x)
\end{gathered}
$$

(cf. [6, §33]). If $\left(\mathrm{D}_{0}\right)$ holds, then in particular $H(t, x, p)>-\infty$ for all $(t, x, p)$, and the closure operations in (2.3) and (2.4) can be omitted. The closure operations can also be omitted if $\left(\mathrm{C}_{0}\right)$ holds, in which event $H(t, x, p)<\infty$ for all $(t, x, p)$.

We consider first the case where $L$ is independent of $t$, because this case is much simpler, and it motivates the more general results which follow.

A convex function $f$ on $R^{n}$ is said to be cofinite if $f$ is the conjugate of a convex function which is everywhere finite on $R^{n}$. This is true if and only if $f$ is lower semicontinuous and proper (i.e. nowhere $-\infty$ and not identically $+\infty$ ), and $f$ satisfies a growth condition of the form

$$
f(v) \geqq \eta(|v|) \text { for all } v \in R^{n},
$$

where $\eta$ is a nondecreasing function from $[0,+\infty)$ to $(-\infty,+\infty]$ such that $\lim _{\lambda \rightarrow+\infty} \eta(\lambda) / \lambda=+\infty$. (This may be seen from [6, Corollary 13.3.1].)

Proposition 1. Suppose that $L$ is independent of $t$.

(a) In order that $\left(\mathrm{C}_{0}\right)$ hold, it is necessary and sufficient that $L(x, \cdot)$ be cofinite for every $x \in R^{n}$ such that $L(x, \cdot)$ is not identically $+\infty$, or equivalently

$$
H(x, p)<+\infty \text { for all }(x, p) \in R^{n} \times R^{n} .
$$

(b) In order that $\left(\mathrm{D}_{0}\right)$ hold, it is necessary and sufficient that $L(x, \cdot)$ not be identically $+\infty$ for any $x \in R^{n}$, or equivalently

$$
H(x, p)>-\infty \text { for all }(x, p) \in R^{n} \times R^{n} .
$$

Proof. Assertion (b) is an immediate consequence of $\left(D_{0}\right)$ and the fact that the convex functions $L(x, \cdot)$ and $H(x, \cdot)$ are conjugate to each other. Assertion (a) is obtained by applying (a) to $M$ and $H$ and using (2.4). The inequality (2.5) says, in view of the convexity of $H(x, \cdot)$, that $H(x, \cdot)$ is for each $x$ either finite everywhere or identically $-\infty$.

COROLLARY. If $L$ is independent of $t$, the following statements are equivalent.

(a) $\left(\mathrm{C}_{0}\right)$ and $\left(\mathrm{D}_{0}\right)$ both hold.

(b) The convex function $L(x, \cdot)$ is cofinite for every $x \in R^{n}$.

(c) The convex function $M(p, \cdot)$ is cofinite for every $p \in R^{n}$.

(d) $H$ is finite on $R^{n} \times R^{n}$.

We now turn to the general case.

Proposition 2. (a) Let $X$ be any open convex subset of $R^{n}$ sufficiently large that condition (D) can be satisfied with a function $\bar{x}$ whose range lies in a compact subset of $X\left(\right.$ e.g. $\left.X=R^{n}\right)$. Then $\left(\mathrm{C}_{0}\right)$ holds if and only if for each $p \in R^{n}$ there exists a 
real-valued function $\gamma_{p}$ on $[0, T] \times X$ such that $\gamma_{p}(t, x)$ is summable in $t \in[0, T]$, concave in $x \in X$, and

$$
H(t, x, p) \leqq \gamma_{p}(t, x), \quad \forall(t, x) \in[0, T] \times X,
$$

or equivalently

$$
L(t, x, v) \geqq\langle v, p\rangle-\gamma_{p}(t, x), \quad \forall(t, x, v) \in[0, T] \times X \times R^{n} .
$$

(b) Let $P$ be any open convex subset of $R^{n}$ sufficiently large that condition (C) can be satisfied with a function $\bar{p}$ whose range lies in a compact subset of $P\left(\right.$ e.g. $\left.P=R^{n}\right)$. Then $\left(\mathrm{D}_{0}\right)$ holds if and only if for each $x \in R^{n}$ there exists a real-valued function $\gamma_{x}$ on $[0, T] \times P$ such that $\gamma_{x}(t, p)$ is summable in $t \in[0, T]$, convex in $p \in P$, and

$$
H(t, x, p) \geqq \gamma_{x}(t, p), \quad \forall(t, p) \in[0, T] \times P,
$$

or equivalently

$$
M(t, p, s) \geqq\langle x, s\rangle+\gamma_{x}(t, p), \quad \forall(t, p, s) \in[0, T] \times P \times R^{n} .
$$

Proof. It suffices by duality to prove (b), since (a) can be obtained by applying (b) to the Hamiltonian $\tilde{H}$ corresponding to $M$ and using (2.3). If $\left(\mathrm{D}_{0}\right)$ holds, then for each $x$ we may actually choose $\gamma_{x}$ to be of the form

$$
\gamma_{x}(t, p)=\langle v(t), p\rangle-\beta(t), \quad v \in L_{n}^{1}, \quad \beta \in L_{1}^{1} .
$$

Thus $\left(D_{0}\right)$ is at least as strong as the condition given in (b).

Conversely, suppose that the condition given in (b) is satisfied. Fix any $x \in R^{n}$, and let $\gamma_{x}$ be a function on $[0, T]$ with the properties described. The inequality (2.9) implies in particular that the convex function $H(t, x, \cdot)$ nowhere has the value $-\infty$. Since $H(t, x, \cdot)$ and $L(t, x, \cdot)$ are convex functions conjugate to each other, and $L(t, x, \cdot)$ likewise does not take on $-\infty$, it follows that neither of these functions is identically $+\infty$.

Let $W$ be a compact subset of $P$ containing the range of $\bar{p}$, where $\bar{p}$ is a function in $L_{n}^{\infty}$ such that $(\mathrm{C})$ is satisfied for certain functions $\bar{s} \in L_{n}^{1}$ and $\bar{\alpha} \in L_{1}^{1}$. Observe that

$$
H(t, x, \bar{p}(t)) \leqq \bar{\alpha}(t)-\langle x, \bar{s}(t)\rangle, \quad \forall t \in[0, T] .
$$

Let $\varepsilon>0$ be sufficiently small that $W+2 \varepsilon B \subset P$, where $B$ is the (closed) unit Euclidean ball in $R^{n}$. Let $U$ be the convex hull of the set $p_{0}-W-\varepsilon B$, where $p_{0}$ is a fixed but arbitrary element of $W$. Then $U$ is a compact, convex neighborhood of 0 such that

$$
W \subset p-U \subset P \text { whenever }\left|p-p_{0}\right| \leqq \varepsilon .
$$

Define $h_{x}$ on $[0, T] \times R^{n}$ by

$$
h_{x}(t, p)=\min _{u \in U} H(t, x, p-u) \leqq H(t, x, p) .
$$

Here $h_{x}(t, \cdot)$ is for each $t$ a lower semicontinuous, convex function from $R^{n}$ to $R^{1} \cup\{+\infty\}$, not identically $+\infty$, because $H(t, x, \cdot)$ is such a function and $U$ is a 
compact convex set; in fact $h_{x}(t, \cdot)$ is obtained by infimal convolution of $H(t, x, \cdot)$ and the indicator $\delta_{U}$ of $U\left[6\right.$, p. 77]. Denoting the conjugate of $h_{x}(t, \cdot)$ by $h_{x}^{*}(t, \cdot)$, we have

$$
h_{x}^{*}(t, v)=L(t, x, v)+\delta_{U}^{*}(v) \geqq L(t, x, v),
$$

where $\delta_{U}^{*}$ is the (finite) support function of $U$. Therefore $h_{x}^{*}$ is a normal convex integrand on $[0, T] \times R^{n}$ by (B) and [8, Corollaries 4.2 and 4.5], and consequently $h_{x}$ itself is a normal convex integrand, since normality is preserved under duality $[7, \mathrm{I}]$.

To establish $\left(\mathrm{D}_{0}\right)$, we need only show, in view of $(2.14)$, that $h_{x}^{*}(t, v(t))$ is summable in $t$ for some $v \in L_{n}^{1}$, and for this it suffices by Theorem 2 of [7, II] to show that $h_{x}(t, p)$ is summable in $t$ for each $p \in R^{n}$ such that $\left|p-p_{0}\right| \leqq \varepsilon$.

From (2.12) and the choice of $W$, we have

$$
h_{x}(t, p) \leqq \inf _{w \in W} H(t, x, w) \leqq H(t, x, \bar{p}(t)),
$$

if $\left|p-p_{0}\right| \leqq \varepsilon$. Thus, by (2.11),

$$
h_{x}(t, p) \leqq \alpha(t) \quad \text { whenever }\left|p-p_{0}\right| \leqq \varepsilon,
$$

where $\alpha$ is the summable function given by

$$
\alpha(t)=\bar{\alpha}(t)-\langle x, \bar{s}(t)\rangle .
$$

On the other hand, if $\left|p-p_{0}\right| \leqq \varepsilon$ we have

$$
h_{x}(t, p) \geqq \inf _{u \in U} \gamma_{x}(t, p-u),
$$

where the infimum is well defined because $p-U \subset P$ by (2.12). Let $\left\{p_{1}, \ldots, p_{k}\right\}$ be a finite subset of $P$ whose convex hull includes $p-U$. (Such a subset exists, because $p-U$ is compact and $P$ is open; see [6, Theorem 20.4].) Let

$$
\beta_{0}(t)=\max \left\{\gamma_{x}\left(t, p_{1}\right), \ldots, \gamma_{x}\left(t, p_{k}\right), 0\right\} \geqq 0 .
$$

Since each of the functions $\gamma_{x}\left(\cdot, p_{k}\right)$ is summable by the assumptions on $\gamma_{x}$, the function $\beta_{0}$ is also summable. Furthermore, the convexity of $\gamma_{x}(t, \cdot)$ implies that $\gamma_{x}\left(t, p^{\prime}\right) \leqq \beta_{0}(t)$ for every $p^{\prime}$ in the convex hull of $\left\{p_{1}, \ldots, p_{k}\right\}$, and thus in particular for every $p^{\prime} \in p-U$.

Now choose $\rho>0$ sufficiently small that $\rho B \subset U \subset \rho^{-1} B$. If $u$ is an arbitrary element of $U, u \neq 0$, we have

$$
p=(1-\lambda) p^{\prime}+\lambda(p-u), \quad 0<\lambda<1, \quad p^{\prime} \in p-U,
$$

where

$$
p^{\prime}=p+\rho|u|^{-1} u, \quad \lambda=\rho /(|u|+\rho) .
$$


The convexity of $\gamma_{x}(t, \cdot)$ then implies that

$$
\gamma_{x}(t, p) \leqq(1-\lambda) \gamma_{x}\left(t, p^{\prime}\right)+\lambda \gamma_{x}(t, p-u),
$$

where

$$
(1-\lambda) \gamma_{x}\left(t, p^{\prime}\right) \leqq(1-\lambda) \beta_{0}(t) \leqq \beta_{0}(t)
$$

and consequently

$$
\gamma_{x}(t, p-u) \geqq \lambda^{-1}\left[\gamma_{x}(t, p)-\beta_{0}(t)\right] \geqq\left[\rho /\left(\rho^{-1}+\rho\right)\right]^{-1}\left[\gamma_{x}(t, p)-\beta_{0}(t)\right] .
$$

Denote the last expression by $\beta(t)$. Then $\beta$ is a summable function such that

$$
\gamma_{x}(t, p-u) \geqq \beta(t), \quad \forall u \in U,
$$

and it follows from (2.16) that $h_{x}(t, p) \geqq \beta(t)$. Since $p$ was any element of $R^{n}$ satisfying $\left|p-p_{0}\right| \leqq \varepsilon$, and (2.15) holds, we may conclude as desired that $h_{x}(t, p)$ is summable in $t$ whenever $\left|p-p_{0}\right| \leqq \varepsilon$.

Corollary 1. Let $X$ be as in Proposition 2(a). Condition $\left(\mathrm{C}_{0}\right)$ is satisfied if there exists an extended-real-valued function $L^{0}$ on $[0, T] \times X \times R^{n}, L^{0} \leqq L$, such that $L^{0}(t, x, v)$ is convex in $(x, v)$ for every $t$, and the function $H^{0}$ on $[0, T] \times X \times R^{n}$ obtained from $L^{0}$ by formula (1.14) is finite and summable in $t \in[0, T]$ for every $(x, p) \in X \times R^{n}$.

Proof. Since $L^{0} \leqq L$ on $[0, T] \times X \times R^{n}$, we have $H^{0} \geqq H$ on $[0, T] \times X \times R^{n}$. Moreover, the convexity of $L^{0}$ in $(x, v)$ implies that $H^{0}(t, x, p)$ is concave in $x$, as well as convex in $p$ [6, Theorem 33.1]. Thus, the condition in Proposition 2(a) is satisfied with $\gamma_{p}(t, x)=H^{0}(t, x, p)$ on $[0, T] \times X$ for each $p$.

Corollary 2. Let $X$ be as in Proposition 2(a). Condition $\left(\mathrm{C}_{0}\right)$ is satisfied in particular if

$$
\begin{array}{r}
L(t, x, v) \geqq f(t, x)+\eta(|v-A(t) x-c(t)|)+\langle v, d(t)\rangle \\
\quad \text { for every }(t, x, v) \in[0, T] \times X \times R^{n},
\end{array}
$$

where the components of $c(t)$ and the matrix $A(T)$ are summable in $t$, the components of $d(t)$ are bounded and measurable in $t, f$ is a real-valued function on $[0, T] \times X$ such that $f(t, x)$ is summable in $t$ and convex in $x$, and $\eta$ is a nondecreasing function from $[0,+\infty)$ to $(-\infty,+\infty]$ such that

$$
\lim _{\lambda \rightarrow \infty} \eta(\lambda) / \lambda=+\infty
$$

(Here any of the functions $A, c, d$ and $f$ could vanish identically.)

Proof. Replacing $\eta$ if necessary by its convex hull, which has the same properties, we can assume without loss of generality that $\eta$ is convex. Denote the expression 
on the right in (2.17) by $L^{0}(t, x, v)$. Then $L^{0}$ is convex in $(x, v)$, and the corresponding Hamiltonian $H^{0}$ on $[0, T] \times X \times R^{n}$ is given by

$$
\begin{aligned}
H^{0}(t, x, p) & =\sup _{v \in R^{n}}\left\{\langle v, p\rangle-L^{0}(t, x, v)\right\} \\
& =-f(t, x)+\sup _{v \in R^{n}}\{\langle v, p-d(t)\rangle-\eta(|v-A(t) x-c(t)|)\} \\
& =-f(t, x)+\sup _{u \in R^{n}}\{\langle u+A(t) x+c(t), p-d(t)\rangle-\eta(|u|)\} \\
& =-f(t, x)+\left\langle A(t) x+c\left(t^{\prime}\right), p-d(t)\right\rangle+\eta^{+}(|p-d(t)|)
\end{aligned}
$$

(cf. [6, Theorem 15.3]), where

$$
\eta^{+}(\mu)=\sup _{\lambda \geqq 0}\{\lambda \mu-\eta(\lambda)\}, \quad \mu \geqq 0 .
$$

The growth condition on $\eta$ implies that $\eta^{+}$is finite (and nondecreasing). Therefore $H^{0}(t, x, p)$ is finite and summable in $t$ for every $(x, p) \in X \times R^{n}$, and we may apply Corollary 1.

REMARK. Trivially, the condition in Corollary 2 is satisfied if

$$
L(t, x, v) \geqq L^{1}(t, x, v)+\eta(|v-A(t) x-c(t)|),
$$

where $\eta, A(t)$ and $c(t)$ have the stated properties and $L^{1}$ satisfies (C).

Proposition 3. Condition $\left(\mathrm{D}_{0}\right)$ implies the following (seemingly much stronger) property: given any bounded set $X \subset R^{n}$, there exist $n \times n$ matrices $B(t)$, vectors $b(t) \in R^{n}$ and scalars $\beta(t)$, all summable with respect to $t$, such that whenever $x$ is $a$ measurable function from $[0, T]$ to $X$, one has

$$
L(t, x(t), v(t)) \leqq \beta(t) \text { for } v(t)=B(t) x(t)+b(t) \text { (summable). }
$$

Proof. Let $S$ be an $n$-dimensional simplex in $R^{n}$ containing $X$, and let $x_{0}, x_{1}, \ldots, x_{n}$ be the vertices of $S$. Condition $\left(\mathrm{D}_{0}\right)$ enables us to find functions $v_{i} \in L_{n}^{1}$ and $\beta_{i} \in L_{1}^{1}(i=0, \ldots, n)$ such that $L\left(t, x_{i}, v_{i}(t)\right) \leqq \beta_{i}(t)$. For each $t \in[0, T]$, let $B(t)$ and $b(t)$ be the unique $n \times n$ matrix and vector in $R^{n}$ such that

$$
v_{i}(t)=B(t) x_{i}+b(t), \quad i=0,1, \ldots, n,
$$

and let

$$
\beta(t)=\max _{i=0, \ldots, n} \beta_{i}(t) .
$$

Then $B(t), b(t)$ and $\beta(t)$ are summable in $t$. Suppose now that $x$ is any measurable function from $[0, T]$ to $X$, and let $v(t)=B(t) x(t)+b(t)$. Then $v \in L_{n}^{1}$. Furthermore, since $x(t) \in S$ there exist unique scalars $\lambda_{i}(t) \geqq 0$ such that

$$
\lambda_{0}(t) x_{0}+\cdots+\lambda_{n}(t) x_{n}=x(t), \quad \lambda_{0}(t)+\cdots+\lambda_{n}(t)=1 .
$$

Relation (2.21) implies that

$$
\lambda_{0}(t) v_{0}(t)+\cdots+\lambda_{n}(t) v_{n}(t)=v(t) .
$$


Thus by the convexity of $L(t, \cdot, \cdot)$ we have

$$
L(t, x(t), v(t)) \leqq \sum_{i=0}^{n} \lambda_{i}(t) L\left(t, x_{i}(t), v_{i}(t)\right) \leqq \beta(t),
$$

as claimed.

REMARK. Proposition 3, or rather its proof, shows that $\left(D_{0}\right)$ is also equivalent to the following, seemingly weaker property: there exists a function $\bar{x} \in L_{n}^{\infty}$ such that for every $y \in R^{n}$ one can find functions $v \in L_{n}^{1}$ and $\beta \in L_{1}^{1}$ with $L(t, \bar{x}(t)+y, v(t))$ $\leqq \beta(t)$. (The latter property implies the property in Proposition 3 by an easy extension of the same argument.)

The next proposition gives other strong forms of $\left(C_{0}\right)$ and $\left(D_{0}\right)$.

Proposition 4. (a) Condition $\left(\mathrm{C}_{0}\right)$ holds if and only if for each bounded set $P \subset R^{n}$ there is a function $L^{0}$ from $[0, T] \times R^{n} \times R^{n}$ to $R^{1} \cup\{+\infty\}$ satisfying (A), (B), (C) and (D), such that $L^{0} \leqq L$, and the function $H^{0}$ obtained from $L^{0}$ by formula (1.14) is finite and summable in $t \in[0, T]$ for every $(x, p) \in R^{n} \times P$.

(b) Condition $\left(\mathrm{D}_{0}\right)$ holds if and only if for each bounded set $X \subset R^{n}$ there is a function $L^{0}$ from $[0, T] \times R^{n} \times R^{n}$ to $R^{1} \cup\{+\infty\}$ satisfying (A), (B), (C) and (D), such that $L^{0} \geqq L$, and the function $H^{0}$ obtained from $L^{0}$ by formula (1.14) is finite and summable in $t \in[0, T]$ for every $(x, p) \in X \times R^{n}$.

Proof. It suffices by duality to prove (b). Suppose that the stated condition is satisfied; we shall show that $\left(\mathrm{D}_{0}\right)$ holds. Given any $\bar{x} \in R^{n}$, let $X=\{\bar{x}\}$ and select a function $L^{0}$ of the type described. The function $f(t, p)=H^{0}(t, \bar{x}, p)$ is then finite and summable in $t \in[0, T]$, as well as convex in $p \in R^{n}$. Therefore by [7, II, Theorem 2] there exists a function $v \in L_{n}^{1}$ such that $f^{*}(t, v(t))$ is summable in $t$, where $f^{*}(t, \cdot)$ is for each $t$ the conjugate of $f(t, \cdot)$. We have

$$
f^{*}(t, v(t))=L^{0}(t, \bar{x}, v(t)) \geqq L(t, \bar{x}, v(t)),
$$

so that $\left(\mathrm{D}_{0}\right)$ is obtained by setting $\beta(t)=f^{*}(t, v(t))$.

Conversely, suppose that $\left(\mathrm{D}_{0}\right)$ holds. In proving that this implies the condition in (b), we can limit attention to the case where $X$ is a nonempty, compact, convex set. For such a set $X$ we may take elements $B(t), b(t)$ and $\beta(t)$ with the properties in Proposition 3 and define

$$
\begin{aligned}
L^{0}(t, x, v) & =\beta(t) \quad \text { if } x \in X \text { and } v=B(t) x+b(t) \\
& =+\infty \text { otherwise. }
\end{aligned}
$$

Then $L^{0}$ satisfies (A), (C) and (D) trivially. It also satisfies (B), as may be seen by a direct application of the definition of "normal convex integrand." (If $Z$ is any countable dense subset of $X$, the countable collection of functions from $[0, T]$ to $R^{n} \times R^{n}$ of the form

$$
t \rightarrow(x(t), v(t))=(z, B(t) z+b(t)), \quad z \in Z,
$$


meets the requirements of the definition.) Furthermore, we have $L^{0} \geqq L$ by (2.20). For any $x \in X$ we have

$$
H^{0}(t, x, p)=\langle B(t) x+b(t), p\rangle-\beta(t),
$$

so that $H^{0}(t, x, p)$ is summable in $t$. Thus $L^{0}$ has the properties specified in the condition in (b).

Corollary. Conditions $\left(\mathrm{C}_{0}\right)$ and $\left(\mathrm{D}_{0}\right)$ both hold if and only if $H(t, x, p)$ is a finite, summable function of $t \in[0, T]$ for every $(x, p) \in R^{n} \times R^{n}$.

REMARK. If $H$ is any real-valued function on $[0, T] \times R^{n} \times R^{n}$ such that $H(t, x, p)$ is summable in $t$, concave in $x$, and convex in $p$, then $H$ is the Hamiltonian corresponding to the function $L$ given by (2.1) [6, Theorem 33.1]. Then $L$ satisfies not only $(A)$ and $(B)$, but also $\left(C_{0}\right)$ and $\left(D_{0}\right)$ by the preceding corollary. (The fact that $L$ satisfies (B) can be seen from the representation

$$
L(t, x, v)=\sup _{1 \leqq k<\infty}\left\{\left\langle v, p_{k}\right\rangle-H\left(t, x, p_{k}\right)\right\},
$$

where $\left\{p_{1}, p_{2}, \ldots\right\}$ is a countable, dense subset of $R^{n}$. For each index $k$, the function

$$
L_{k}(t, x, v)=\left\langle v, p_{k}\right\rangle-H\left(t, x, p_{k}\right)
$$

is finite, convex in $(x, v)$ and measurable in $t$, and consequently $L_{k}$ is a normal convex integrand [7, I, Lemma 2]. Since $L$ is the pointwise supremum of the functions $L_{k}$ by (2.22), $L$ is itself a normal convex integrand [8, Corollary 4.1].)

3. Perturbations of the Bolza functionals. The duality between the problem of minimizing $\Phi_{l, L}$ and the problem of minimizing $\Phi_{m, M}$ has been explained in $[9, \S 8]$ in terms of certain perturbations of $\Phi_{l, L}$ and $\Phi_{m, M}$. A more detailed study of such perturbations yields, not only Theorem 1, but also, as shown in $\$ 5$, a dual interpretation of optimal arcs for either problem in terms of "Lagrange multipliers" for the other.

As in [9], we identify the dual $A_{n}^{1 *}$ of the Banach space $A_{n}^{1}$ with $R^{n} \oplus L_{n}^{\infty}$ under the pairing

$$
\langle(a, y), p\rangle=\langle a, p(0)\rangle+\int_{0}^{T}\langle y(t), \dot{p}(t)\rangle d t,
$$

the norm being

$$
\|(a, y)\|=\max \left\{|a|,\|y\|_{\infty}\right\} .
$$

For each $a \in R^{n}$ and $y \in L_{n}^{\infty}$ we denote by $\varphi_{l, L}(a, y)$ the infimum of

$$
l(x(0)+a, x(T))+\int_{0}^{T} L(t, x(t)+y(t), \dot{x}(t)) d t
$$

over all arcs $x \in A_{n}^{1}$. The extended-real-valued function $\varphi_{l, L}$ on $A_{n}^{1 *}$ is well defined and convex, and the function $\varphi_{l, L}^{*}$ on $A_{n}^{1}$ conjugate to $\varphi_{l, L}$ is $\Phi_{m, M}[9$, Theorem 3]. 
Similarly, for each $b \in R^{n}$ and $q \in L_{n}^{\infty}$ we denote by $\varphi_{m, M}(b, q)$ the infimum of

$$
m(p(0)+b, p(t))+\int_{0}^{T} M(t, p(t)+q(t), \dot{p}(t)) d t
$$

over all arcs $p \in A_{n}^{1}$, and then $\varphi_{m, M}$ is a well-defined convex functional on $A_{n}^{1 *}$ whose conjugate on $A_{n}^{1}$ is $\Phi_{l, L}$. One has

$$
\begin{aligned}
\inf _{x \in A_{n}^{1}} \Phi_{l, L}(x) & =\varphi_{l, L}(0,0), \\
\inf _{p \in A_{n}^{1}} \Phi_{m, M}(p) & =\varphi_{m, M}(0,0) .
\end{aligned}
$$

Furthermore, unless $\Phi_{l, L}$ and $\Phi_{m, M}$ are both identically $+\infty$ on $A_{n}^{1}$ and the origin of $A_{n}^{1 *}$ does not belong to the weak* closure of either dom $\varphi_{l, L}$ or dom $\varphi_{m, M}$, one has

$$
\begin{aligned}
& -\inf _{x \in A_{n}^{1}} \Phi_{m, M}(p)=\text { weak }^{*} \liminf _{(a, y) \rightarrow(0,0)} \varphi_{l, L}(a, y), \\
& -\inf _{x \in A_{n}^{1}} \Phi_{l, L}(x)=\text { weak }^{*} \liminf _{(b, q) \rightarrow(0,0)} \varphi_{m, M}(b, q) .
\end{aligned}
$$

It is clear from these facts that the equality of the extrema in Theorem 1 can be established by showing that either $\varphi_{l, L}$ or $\varphi_{m, M}$ is weak* lower semicontinuous at the origin of $A_{n}^{1 *}$ under the stated conditions. Our pattern of proof is as follows. Assuming condition $\left(\mathrm{D}_{0}\right)$, we show in this section that the weak* topology on $A_{n}^{1 *}$ can be replaced by the strong topology in (3.7), and that there exist points of the convex set

$$
\operatorname{dom} \varphi_{l, L}=\left\{(a, y) \in A_{n}^{1 *} \mid \varphi_{l, L}(a, y)<+\infty\right\}
$$

at which $\varphi_{l, L}$ is not only strongly lower semicontinuous, but actually strongly continuous relative to aff $\left(\operatorname{dom} \varphi_{l, L}\right)$. In $\S 4$, we show that the origin is such a point if and only if ri $C_{l} \cap$ ri $C_{L} \neq \varnothing$.

While the equality of the extrema in Theorem 1 may be deduced from special lower semicontinuity properties of $\varphi_{l, L}$ and $\varphi_{m, M}$, the attainment of these extrema may be deduced from differentiability properties. The study of such differentiability properties also leads to a dual interpretation of optimal arcs in convex problems of Bolza, as we explain in $\$ 5$.

Recall that a subgradient of $\varphi_{l, L}$ at a point $(\bar{a}, \bar{y})$ in $A_{n}^{1 *}$ is an element $p$ of the space $A_{n}^{1 * *}$ such that

$$
\varphi_{l, L}(a, y) \geqq \varphi_{l, L}(\bar{a}, \bar{y})+\langle(a, y)-(\bar{a}, \bar{y}), p\rangle, \quad \forall(a, y) \in A_{n}^{1 *} .
$$

Here $\langle\cdot, \cdot\rangle$ denotes the canonical pairing between $A_{n}^{1 *}$ and $A_{n}^{1 * *}$; if $p$ belongs to $A_{n}^{1}$, regarded as a subspace of $A_{n}^{1 * *}$, this pairing is expressed by (3.1). The set of all subgradients of $\varphi_{l, L}$ at $(\bar{a}, \bar{y})$ is denoted by $\partial \varphi_{l, L}(\bar{a}, \bar{y})$. This is a closed convex subset of $A_{n}^{1 * *}$.

If $\varphi_{l, L}(\bar{a}, \bar{y})=+\infty$, the set $\partial \varphi_{l, L}(\bar{a}, \bar{y})$ is empty, whereas if $\varphi_{l, L}(\bar{a}, \bar{y})=-\infty$, it is all of $A_{n}^{1 * *}$. If $\varphi_{l, L}(\bar{a}, \bar{y})$ is finite, the elements of $\partial \varphi_{l, L}(\bar{a}, \bar{y})$ can be described in terms 
of directional derivatives of $\varphi_{l, L}$ (see $\S 5$ ). They correspond to the "nonvertical" closed supporting hyperplanes to the epigraph of $\varphi_{l, L}$, which is the set

$$
\text { epi } \varphi_{l, L}=\left\{(a, y, \mu) \in A_{n}^{1 *} \oplus R^{1} \mid \mu \geqq \varphi_{l, L}(a, y)\right\},
$$

at the point $(\bar{a}, \bar{y}, \bar{\mu})$, where $\bar{\mu}=\varphi_{l, L}(\bar{a}, \bar{y})$. Thus the existence of subgradients can be deduced from standard theorems about the existence of supporting hyperplanes.

The significance of subgradients for problems of Bolza is apparent from the following consequence of Theorem 3 of [9].

Proposition 5. An arc $p \in A_{n}^{1}$ belongs to $\partial \varphi_{l, L}(0,0)$ if and only if it satisfies

$$
\Phi_{m, M}(p)=\inf _{A_{n}^{1}} \Phi_{m, M}=-\inf _{A_{n}^{1}} \Phi_{l, L}
$$

Dually, an arc $x \in A_{n}^{1}$ belongs to $\partial \varphi_{m, M}(0,0)$ if and only if it satisfies

$$
\Phi_{l, L}(x)=\inf _{A_{n}^{1}} \Phi_{l, L}=-\inf _{A_{n}^{1}} \Phi_{m, M} .
$$

Proof. The definition of "subgradient" implies that $p$ belongs to $\partial \varphi_{l, L}(0,0)$ if and only if

$$
\begin{aligned}
-\varphi_{l, L}(0,0) & \geqq \sup \left\{\langle(a, y), p\rangle-\varphi_{l, L}(a, y) \mid(a, y) \in A_{n}^{1 *}\right\} \\
& =\varphi_{l, L}^{*}(p)=\Phi_{m, M}(p) .
\end{aligned}
$$

This inequality is equivalent to (3.12), in view of (1.13) and (3.5). The dual assertion is proved similarly.

In the study of continuity and the existence of subgradients, the following known facts (which hold for any convex function) are basic. There exist points of dom $\varphi_{l, L}$ at which $\varphi_{l, L}$ is (strongly) continuous relative to aff (dom $\varphi_{l, L}$ ) if and only if epi $\varphi_{l, L}$ has a nonempty relative interior. If the latter is true, then the points of dom $\varphi_{l, L}$ at which $\varphi_{l, L}$ is continuous relative to aff $\left(\operatorname{dom} \varphi_{l, L}\right)$ are precisely the points of ri (dom $\varphi_{l, L}$ ), which is the projection of ri (epi $\left.\varphi_{l, L}\right)$ on $A_{n}^{1 *}$. Furthermore, then $\varphi_{l, L}$ is subdifferentiable at every point $(a, y)$ of $\mathrm{ri}\left(\operatorname{dom} \varphi_{l, L}\right)$, i.e. the set $\partial \varphi_{l, L}(a, y)$ is nonempty in $A_{n}^{1 * *}$. These facts are usually stated in terms of continuity relative to the whole space, but the generalization to the case of continuity relative to aff (dom $\varphi_{l, L}$ ) is obvious. (In proving the subdifferentiability assertion, one uses the Hahn-Banach Theorem to extend a continuous affine function on aff (dom $\varphi_{l, L}$ ) majorized by $\varphi_{l, L}$ to a continuous affine function on all of $A_{n}^{1 *}$.)

The usual limitation of the facts just cited, if they can be applied at all, is that they lead to conclusions involving $A_{n}^{1 * *}$, rather than $A_{n}^{1}$. In particular, they only furnish the existence of subgradients of $\varphi_{l, L}$ in $A_{n}^{1 * *}$, whereas, if Proposition 5 is to be of use, one needs the existence of subgradients in $A_{n}^{1}$. The chief virtue of condition $\left(D_{0}\right)$ is that it enables one to surmount this difficulty.

THEOREM 2. Assume that $\left(\mathrm{D}_{0}\right)$ holds. Then the conjugate convex function $\varphi_{l, L}^{*}$ $=\Phi_{m, M}^{* *}$ on $A_{n}^{1 * *}$ is just $\Phi_{m, M}$ on $A_{n}^{1}$, in the sense that it agrees with $\Phi_{m, M}$ on $A_{n}^{1}$ (the 
latter space regarded canonically as a subspace of $A_{n}^{1 * *}$ ), whereas it has the value $+\infty$ everywhere outside of $A_{n}^{1}$.

Furthermore, then aff (dom $\left.\varphi_{l, L}\right)$ is closed and of finite codimension in $A_{n}^{1 *}$, ri (dom $\left.\varphi_{l, L}\right)$ is nonempty, and $\varphi_{l, L}$ is continuous relative to aff $\left(\operatorname{dom} \varphi_{l, L}\right)$ at every point of ri (dom $\left.\varphi_{l, L}\right)$.

Proof. We deduce the assertion about $\varphi_{l, L}^{*}$ from a more fundamental result in [7, II] for conjugates of integral functionals on the space $L_{n}^{\infty}$. Only the values of $\varphi_{l, L}^{*}$ outside of $A_{n}^{1}$ need to be dealt with, since we already know that $\Phi_{m, M}$ is the function on $A_{n}^{1}$ conjugate to $\varphi_{l, L}$ with respect to the pairing between $A_{n}^{1}$ and $A_{n}^{1 *}$. Each element of $A_{n}^{1 * *} \backslash A_{n}^{1}$ corresponds to a linear functional on $A_{n}^{1 *}$ of the form

$$
(a, y) \rightarrow\langle a, b\rangle+s(y), \quad b \in R^{n}, \quad s \in L_{n}^{\infty *} \mid L_{n}^{1} .
$$

Thus our task is to prove under $\left(\mathrm{D}_{0}\right)$ that, for any $b \in R^{n}$ and $s \in L_{n}^{\infty *} \mid L_{n}^{1}$, one has

$$
\sup \left\{\langle a, b\rangle+s(y)-\varphi_{l, L}(a, y) \mid a \in R^{n}, y \in L_{n}^{\infty}\right\}=+\infty .
$$

According to the definition of $\varphi_{l, L}$ the supremum (3.14) is the same as the supremum of

$$
\langle a, b\rangle+s(y)-l(x(0)+a, x(T))-\int_{0}^{T} L(t, x(t)+y(t), \dot{x}(t)) d t
$$

over all $a \in R^{n}, y \in L_{n}^{\infty}$ and $x \in A_{n}^{1}$, and hence it equals

$$
\begin{aligned}
\sup \left\{\left\langle c_{0}-x(0), b\right\rangle\right. & +s(u-x)-l\left(c_{0}, x(T)\right) \\
& \left.-\int_{0}^{T} L(t, u(t), \dot{x}(t)) d t \mid c_{0} \in R^{n}, u \in L_{n}^{\infty}, x \in A_{n}^{1}\right\} .
\end{aligned}
$$

The continuous linear functional $x \rightarrow\langle x(0), b\rangle+s(x)$ on $A_{n}^{1}$ can also be represented in the form

$$
x \rightarrow\langle x(T), d\rangle-\int_{0}^{T}\langle\dot{x}(t), r(t)\rangle d t
$$

for certain elements $d \in R^{n}$ and $r \in L_{n}^{\infty}$ uniquely determined by $b$ and $s$. Then (3.15) can be re-expressed as

$$
\begin{aligned}
\sup \left\{\left\langle c_{0}, b\right\rangle-\right. & \langle x(T), d\rangle+s(u)+\int_{0}^{T}\langle\dot{x}(t), r(t)\rangle d t \\
& \left.\quad-l\left(c_{0}, x(T)\right)-\int_{0}^{T} L(t, u(t), \dot{x}(t)) d t \mid c_{0} \in R^{n}, u \in L_{n}^{\infty}, x \in A_{n}^{1}\right\} \\
=\sup & \left\{\left\langle c_{0}, b\right\rangle-\left\langle c_{T}, d\right\rangle+s(u)+\int_{0}^{T}\langle v(t), r(t)\rangle d t\right. \\
& \left.\quad-l\left(c_{0}, c_{T}\right)-\int_{0}^{T} L(t, u(t), v(t)) d t \mid\left(c_{0}, c_{T}\right) \in R^{n} \times R^{n}, u \in L_{n}^{\infty}, v \in L_{n}^{1}\right\} \\
= & m(b, d)+\sup _{u \in L_{n}^{\infty}}\left\{s(u)+\sup _{v \in L_{n}^{1}}\left\{\int_{0}^{T}\langle v(t), r(t)\rangle d t-\int_{0}^{T} L(t, u(t), v(t)) d t\right\}\right\} .
\end{aligned}
$$


We claim that the inner supremum in the latter expression is

$$
\int_{0}^{T} H(t, u(t), r(t)) d t
$$

so that our task reduces to showing that

$$
\sup \left\{s(u)+\int_{0}^{T} H(t, u(t), r(t)) d t \mid u \in L_{n}^{\infty}\right\}=+\infty .
$$

To see that the inner supremum equals (3.16), consider (for any fixed $u \in L_{n}^{\infty}$ ) the function

$$
f(t, v)=L(t, u(t), v), \quad(t, v) \in[0, T] \times R^{n} .
$$

Condition $\left(\mathrm{D}_{0}\right)$ implies in particular that $f(t, \cdot)$ is not identically $+\infty$ on $R^{n}$ for any $t \in[0, T]$, and it follows then from [8, Corollary 4.5] and conditions (A) and (B) on $L$ that $f$ is a normal convex integrand on $[0, T] \times R^{n}$ in the sense of [7], [8]. The normal convex integrand conjugate to $f$ is

$$
f^{*}(t, p)=H(t, u(t), p)
$$

by (1.14). There exist by Proposition 3 functions $\bar{v} \in L_{n}^{1}$ and $\beta \in L_{1}^{1}$ such that $L(t, u(t), \bar{v}(t)) \leqq \bar{\beta}(t)$. On the other hand, since (C) holds there exist functions $\bar{p} \in L_{n}^{\infty}, \bar{s} \in L_{n}^{1}$ and $\bar{\alpha} \in L_{1}^{1}$ such that

We then have

$$
L(t, u(t), v) \geqq\langle u(t), \bar{s}(t)\rangle+\langle v, \bar{p}(t)\rangle-\bar{\alpha}(t) .
$$

and

$$
H(t, u(t), p) \geqq\langle\bar{v}(t), p\rangle-\bar{\beta}(t)
$$

$$
H(t, u(t), \bar{p}(t)) \leqq \bar{\alpha}(t)-\langle u(t), \bar{s}(t)\rangle
$$

by (1.14). Thus $f(t, \bar{v}(t))$ and $f^{*}(t, \bar{p}(t))$ are summable in $t$. This implies by [7, I, Theorem 2] that the functionals

$$
\begin{array}{ll}
I_{f}(v)=\int_{0}^{T} f(t, v(t)) d t=\int_{0}^{T} L(t, u(t), v(t)) d t, & v \in L_{n}^{1}, \\
I_{f} \cdot(p)=\int_{0}^{T} f^{*}(t, p(t)) d t=\int_{0}^{T} H(t, u(t), p(t)) d t, & p \in L_{n}^{\infty},
\end{array}
$$

are conjugate to each other, and hence in particular

as claimed.

$$
I_{f} \cdot(r)=\sup \left\{\int_{0}^{T}\langle v(t), r(t)\rangle d t-I_{f}(v) \mid v \in L_{n}^{1}\right\}
$$

We show now by a similar argument that (3.17) holds for any $r \in L_{n}^{\infty}$, assuming of course that $s \in L_{n}^{\infty} * \mid L_{n}^{1}$. As above, let $\bar{p}, \bar{s}$, and $\bar{\alpha}$ be functions satisfying (C), so that

$$
H(t, x, \bar{p}(t)) \leqq \bar{\alpha}(t)-\langle x, \bar{s}(t)\rangle, \quad \forall(t, x) \in[0, T] \times R^{n}
$$


Let $\rho$ be a real number larger than $\|\bar{p}\|_{\infty}$ and $\|r\|_{\infty}$, and define $g$ on $[0, T] \times R^{n}$ by

$$
g(t, x)=-\min \{H(t, x, p)|| p \mid \leqq \rho\} \geqq-H(t, x, r(t)) .
$$

(The minimum is attained, because $H(t, x, p)$ is lower semicontinuous in $p$ by (1.14).) The concavity of $H(t, x, p)$ in $x$ implies that $g(t, x)$ is convex in $x$. Moreover, $g(t, x)$ is measurable in $t$ by [8, Corollary 4.3], because $H(\cdot, x, \cdot)$ is a normal convex integrand on $[0, T] \times R^{n}$ for any $x \in R^{n}$ (take $u(t)=x$ in the above argument concerning (3.18)). In fact $g(t, x)$ is summable in $t$ for each $x$ : we have

$$
g(t, x) \geqq-H(t, x, \bar{p}(t)) \geqq\langle x, \bar{s}(t)\rangle-\bar{\alpha}(t)
$$

by (3.20) and (3.19), while if $v \in L_{n}^{1}$ and $\beta \in L_{1}^{1}$ are functions such that $L(t, x, v(t))$ $\leqq \beta(t)$ (and such functions exist by $\left(\mathrm{D}_{0}\right)$ ) we have

$$
H(t, x, p) \geqq\langle v(t), p\rangle-\beta(t),
$$

so that

$$
g(t, x) \leqq-\inf \{\langle v(t), p\rangle-\beta(t)|| p \mid \leqq \rho\}=\rho|v(t)|+\beta(t) .
$$

Inasmuch as $g(t, x)$ is convex in $x$ and summable in $t$, we may conclude from [7, II, Theorem 2] that the convex functional

$$
I_{g}(u)=\int_{0}^{T} g(t, u(t)) d t
$$

is well defined on $L_{n}^{\infty}$ and that, since $s \in L_{n}^{\infty} * \mid L_{n}^{1}$, we have

$$
\sup _{u \in L_{n}^{\infty}}\left\{s(u)-\int_{0}^{T} g(t, u(t)) d t\right\}=I_{g}^{*}(s)=+\infty .
$$

This yields the desired relation (3.17), because (3.20) implies

$$
-g(t, u(t)) \leqq H(t, u(t), r(t)) .
$$

The second paragraph of Theorem 2 still must be verified. If a convex set $C$ in some locally convex space has the property that aff $C$ is closed and of finite codimension, and ri $C \neq \varnothing$, then every convex set $C^{\prime}$ containing $C$ has the same property. Thus it suffices (by the remarks preceding the theorem) to show that epi $\varphi_{l, L}$ contains a convex set $C$ such that aff $C$ is closed and of finite codimension, and ri $C \neq \varnothing$.

Let $X$ be any bounded subset of $R^{n}$ with a nonempty interior, and let $B(t), b(t)$, and $\beta(t)$ be as in Proposition 3. For each $y \in L_{n}^{\infty}$ let $x_{y}$ denote the unique arc in $A_{n}^{1}$ satisfying the differential equation

$$
\dot{x}_{y}(t)=B(t)\left[x_{y}(t)+y(t)\right], \quad x_{y}(T)=0,
$$

and let

$$
N=\left\{(a, y) \in A_{n}^{1 *} \mid a=-x_{y}(0)\right\} .
$$


Then $N$ is a closed subspace of $A_{n}^{1 *}$ of finite codimension. Choose any $\bar{x} \in$ int $X$ and any $\left(c_{0}, c_{T}\right)$ such that $l\left(c_{0}, c_{T}\right)<+\infty$, and let $\left(a^{0}, y^{0}\right) \in A_{n}^{1 *}$ and $x^{0} \in A_{n}^{1}$ be the unique elements such that

$$
\begin{aligned}
x^{0}(0)+a^{0} & =c_{0}, & & x^{0}(T)=c_{T}, \\
x^{0}(t)+y^{0}(t) & =\bar{x}, & & \dot{x}^{0}(t)=B(t) \bar{x}+b(t) .
\end{aligned}
$$

It is possible to choose an $\varepsilon>0$ such that

$$
\left[x_{y}(t)+y(t)+\bar{x}\right] \in X
$$

for almost every $t \in[0, T]$, whenever $\|y\|_{\infty} \leqq \varepsilon$. Then for any $(a, y) \in N$ with $\|y\|_{\infty} \leqq \varepsilon$ we have

$$
\begin{gathered}
l\left(x^{0}(0)+x_{y}(0)+a^{0}+a, x^{0}(T)+x_{y}(T)\right)=l\left(c_{0}, c_{T}\right), \\
L\left(t, x^{0}(t)+x_{y}(t)+y^{0}(t)+y(t), \dot{x}^{0}(t)+\dot{x}_{y}(t)\right) \\
=L\left(t, x_{y}(t)+y(t)+\bar{x}, B(t)\left[x_{y}(t)+y(t)+\bar{x}\right]+b(t)\right) \leqq \beta(t),
\end{gathered}
$$

so that by definition

$$
\varphi_{l, L}\left(a^{0}+a, y^{0}+y\right) \leqq l\left(c_{0}, c_{T}\right)+\int_{0}^{T} \beta(t) d t .
$$

Denote the right side of (3.24) by $\alpha$ (a real number). We then have

$$
\varphi_{l, L}\left(a^{0}+a, y^{0}+y\right) \leqq \alpha \quad \text { whenever }(a, y) \in N,\|y\|_{\infty} \leqq \varepsilon .
$$

Thus epi $\varphi_{l, L}$ contains a translate $C$ of the convex set

$$
\tilde{C}=\left\{(a, y, \mu) \in A_{n}^{1 *} \oplus R^{1} \mid(a, y) \in N,\|y\|_{\infty} \leqq \varepsilon, \mu \geqq 0\right\} .
$$

Obviously aff $\widetilde{C}=N \oplus R^{1}$, which is closed and of finite codimension, and ri $\widetilde{C} \neq \varnothing$. Hence aff $C$ is closed and of finite codimension, and ri $C \neq \varnothing$.

COROLlaRY 1. If $\left(\mathrm{D}_{0}\right)$ holds, then every continuous affine function on $A_{n}^{1 *}$ majorized by $\varphi_{l, L}$ corresponds to an element of $A_{n}^{1}$, rather than a more general element of $A_{n}^{1 * *}$. In particular, for every $(a, y) \in A_{n}^{1 *}$ such that $\varphi_{l, L}(a, y) \neq-\infty$, the subgradient set $\partial \varphi_{l, L}(a, y)$ is actually contained in $A_{n}^{1}$.

Proof. By definition, the continuous linear functions on $A_{n}^{1 *}$ corresponding to affine functions majorized by $\varphi_{l, L}$ are the elements of $A_{n}^{1 * *}$ for which the value of $\varphi_{l, L}^{*}$ is not $+\infty$. Theorem 2 says that there are no such elements other than elements of $A_{n}^{1}$.

COROllary 2. If $\left(\mathrm{D}_{0}\right)$ holds, then every continuous linear function on $A_{n}^{1 *}$ bounded above (or below) on dom $\varphi_{l, L}$ can be identified with an element of $A_{n}^{1}$, rather than a more general element of $A_{n}^{1 * *}$.

Proof. Define $l^{0}$ and $L^{0}$ by

$$
\begin{aligned}
l^{\circ}\left(c_{0}, c_{T}\right) & =\max \left\{l\left(c_{0}, c_{T}\right), 0\right\}, \\
L^{0}(t, x, v) & =\max \{L(t, x, v), 0\} .
\end{aligned}
$$




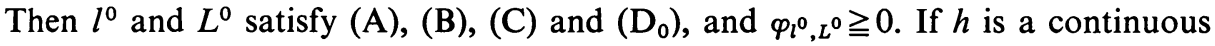
linear function on $A_{n}^{1 *}$ whose supremum $\alpha$ over $\operatorname{dom} \varphi_{l, L}$ is finite, then

$$
\varphi_{l^{0}, L^{0}} \geqq h-\alpha .
$$

Hence by Corollary 1 , applied to $l^{0}$ and $L^{0}, h$ corresponds to an element of $A_{n}^{1}$.

COROllary 3. If $\left(\mathrm{D}_{0}\right)$ holds, then the limit

$$
\liminf _{(a, y) \rightarrow(\bar{a}, \bar{y})} \varphi_{l, L}(a, y)
$$

is the same whether it is taken with respect to the weak* topology or the strong topology on $A_{n}^{1 *}$. In particular, under $\left(\mathrm{D}_{0}\right)$ one has

$$
-\inf _{p \in A_{n}^{1}} \Phi_{m, M}(p)=\text { strong } \liminf _{(a, y) \rightarrow(0,0)} \varphi_{l, L}(a, y),
$$

unless $\Phi_{l, L}$ and $\Phi_{m, M}$ are identically $+\infty$ on $A_{n}^{1}$ and the origin of $A_{n}^{1 *}$ is not in the weak* closure of either $\operatorname{dom} \varphi_{l, L}$ or $\operatorname{dom} \varphi_{m, M}$.

Proof. The limit (3.29) is the same in both topologies for every $(\bar{a}, \bar{y})$ if and only if the closure of the epigraph of $\varphi_{l, L}$ in $A_{n}^{1 *} \oplus R^{1}$ is the same for both topologies. Since the epigraph is convex, its closure for a particular topology is the intersection of the half-spaces which contain it and are closed for that topology. Corollaries 1 and 2 imply that, if $\left(\mathrm{D}_{0}\right)$ holds, the half-spaces in question in $A_{n}^{1 *} \oplus R^{1}$ are the same, whether one is considering $A_{n}^{1 *}$ in the weak* topology or the strong topology. Formula (3.29) follows from (3.7).

Corollary 4. Assume that $\left(\mathrm{D}_{0}\right)$ holds. If $\varphi_{l, L}$ nowhere has the value $-\infty$, then $\Phi_{m, M}$ is not identically $+\infty$, and the conjugate convex function $\Phi_{m, M}^{*}$ on $A_{n}^{1 *}$ agrees with $\varphi_{l, L}$ except perhaps at relative boundary points of dom $\varphi_{l, L}$. On the other hand, if $\varphi_{l, L}$ has the value $-\infty$ somewhere, then $\varphi_{l, L}$ is identically $-\infty$ on $\mathrm{ri}\left(\mathrm{dom} \varphi_{l, L}\right)$, while $\Phi_{m, M}$ is identically $-\infty$ on $A_{n}^{1 *}$ and hence agrees with $\varphi_{l, L}$ on $\mathrm{ri}\left(\mathrm{dom} \varphi_{l, L}\right)$ (but does not agree with $\varphi_{l, L}$ outside of $\operatorname{dom} \varphi_{l, L}$, since there $\varphi_{l, L}$ is identically $+\infty$ ).

Proof. This is obtained from Corollary 3 and the fact in Theorem 2 that aff $\left(\operatorname{dom} \varphi_{l, L}\right)$ is closed, and $\varphi_{l, L}$ is continuous relative to aff (dom $\left.\varphi_{l, L}\right)$ except perhaps at boundary points of dom $\varphi_{l, L}$ relative to aff $\left(\operatorname{dom} \varphi_{l, L}\right)$. In general, since $\Phi_{m, M}$ is the conjugate of $\varphi_{l, L}$ on $A_{n}^{1}, \Phi_{m, M}^{*}(\bar{a}, \bar{y})$ is given by the limit (3.28) unless $(\bar{a}, \bar{y})$ is not in the weak* closure of $\operatorname{dom} \varphi_{l, L}$ and $\Phi_{m, M}$ is identically $+\infty$ [3].

The next two corollaries are major steps in the direction of Theorem 1.

Corollary 5. If $\left(\mathrm{D}_{0}\right)$ holds and $(0,0) \in \mathrm{ri}\left(\operatorname{dom} \varphi_{l, L}\right)$, then

$$
\inf _{x \in A_{n}^{1}} \Phi_{l, L}(x)=-\min _{p \in A_{n}^{1}} \Phi_{m, M}(p)<+\infty .
$$

Proof. Theorem 2 asserts that $\varphi_{l, L}$ is continuous at $(0,0)$ relative to aff (dom $\varphi_{l, L}$ ), and therefore $\partial \varphi_{l, L}(0,0)$ is not empty. In fact $\partial \varphi_{l, L}(0,0)$ is contained in $A_{n}^{1}$ according 
to Corollary 1 , except in the trivial case where $\partial \varphi_{l, L}(0,0)$ is all of $A_{n}^{1 * *}$. Thus $\varphi_{l, L}$ has at least one subgradient $p \in A_{n}^{1}$ at $(0,0)$, and the result follows from Proposition 5.

Corollary 6. Assume that $\left(\mathrm{D}_{0}\right)$ holds, and that $\Phi_{m, M}$ is not identically $+\infty$ on $A_{n}^{1}$. Then each of the closed, convex level sets

$$
\left\{p \in A_{n}^{1} \mid \Phi_{m, M}(p) \leqq \mu\right\}, \quad \mu \in R^{1},
$$

is a locally compact space relative to the weak topology on $A_{n}^{1}$ and in particular has the property that every bounded subset is weakly relatively compact. The sets (3.30) are themselves bounded (and hence weakly compact) if and only if

$$
(0,0) \in \operatorname{int}\left(\operatorname{dom} \varphi_{l, L}\right) \text {. }
$$

Proof. In general, for each real number $\mu$ greater than the infimum of $\varphi_{l, L}$ on $A_{n}^{1}$, the convex level set (3.30), regarded as a subset of $A_{n}^{1 * *}$, has as its closure in the weak** topology (the weak topology induced on $A_{n}^{1 * *}$ by $A_{n}^{1 *}$ ) the corresponding level set of the function $\Phi_{m, M}^{* *}$ on $A_{n}^{1 * *}$. These level sets are the same according to Theorem 2 if $\left(\mathrm{D}_{0}\right)$ holds, so that the set (3.30) is weak** closed in $A_{n}^{1 * *}$. Then bounded subsets of (3.30) are weak** compact in $A_{n}^{1 * *}$, or in other words weakly compact in $A_{n}^{1}$. A basic theorem about convex functionals [11, Theorem 7A] asserts that (3.30) is bounded in $A_{n}^{1}$ for every real $\mu$ if and only if the origin belongs to int (dom $\Phi_{m, M}^{*}$ ) in $A_{n}^{1 *}$. The latter condition is equivalent to (3.31) by Corollary 4 . Thus the set (3.30) is weakly compact for every $\mu$ if and only if (3.31) holds.

If the set (3.30) is not bounded, it is nevertheless "bounded locally" relative to the weak topology and therefore locally weakly compact. This local boundedness property follows by a general argument from the fact that (according to Corollary 4 and the second paragraph of Theorem 2) the convex set

$$
D=\operatorname{dom} \Phi_{m, M}^{*} \subset A_{n}^{1 *}
$$

has aff $D$ closed and of finite codimension, and ri $D \neq \varnothing$; cf. Joly [13].

Here is the argument. Fix any $\mu \in R^{1}$ and $\bar{p} \in A_{n}^{1}$ such that $\Phi_{m, M}(\bar{p}) \leqq \mu$, and denote the set (3.30) by $C$. We construct a weak neighborhood $W$ of $\bar{p}$ such that $W \cap C$ is bounded in $A_{n}^{1}$. Let $J$ be a finite-dimensional subspace of $A_{n}^{1 *}$ complementary to aff $D$. Then each element $(a, y)$ of $A_{n}^{1 *}$ can be represented uniquely in the form

$$
(a, y)=\left(a^{\prime}, y^{\prime}\right)+\left(a^{\prime \prime}, y^{\prime \prime}\right), \quad\left(a^{\prime}, y^{\prime}\right) \in \operatorname{aff} D, \quad\left(a^{\prime \prime}, y^{\prime \prime}\right) \in J,
$$

and the components $\left(a^{\prime}, y^{\prime}\right)$ and $\left(a^{\prime \prime}, y^{\prime \prime}\right)$ depend continuously on $(a, y)$. Using the representation (3.32), we define the convex function $\psi$ on $A_{n}^{1 *}$ by

$$
\psi(a, y)=\Phi_{m, M}^{*}\left(a^{\prime}, y^{\prime}\right)+\alpha\left\|\left(a^{\prime \prime}, y^{\prime \prime}\right)\right\|,
$$


where $\alpha$ is a real number, $\alpha>\|p\|$. The conjugate of $\psi$ on $A_{n}^{1}$ is then given by

$$
\begin{aligned}
\psi^{*}(p) & =\Phi_{m, M}(p) & & \text { if } p \in W_{0}, \\
& =+\infty & & \text { if } p \notin W_{0}
\end{aligned}
$$

where

$$
W_{0}=\left\{p \mid\left\langle p,\left(a^{\prime \prime}, y^{\prime \prime}\right)\right\rangle \leqq \alpha\left\|\left(a^{\prime \prime}, y^{\prime \prime}\right)\right\|, \forall\left(a^{\prime \prime}, y^{\prime \prime}\right) \in J\right\} .
$$

Note that $W_{0}$ is a weak neighborhood of the origin of $A_{n}^{1}$, because $\alpha^{-1} W_{0}$ is the polar of the finite-dimensional compact convex set $J \cap U, U$ being the unit ball of $A_{n}^{1 *}$. Moreover $\bar{p}$ belongs to the strong interior of $W_{0}$, inasmuch as $\alpha>\|\bar{p}\|$, and hence $W_{0}$ is also a weak neighborhood of $\bar{p}$. We now choose any element $(\bar{a}, \bar{y})$ of the (strong) interior of dom $\psi$ in $A_{n}^{1 *}$; such an element exists by the definition of $\psi$, because ri $D \neq \varnothing$. Invoking [11, Theorem $7 \mathrm{~A}]$ as above, we obtain the fact that for every $\bar{\mu} \in R^{1}$ the set

$$
\left\{p \in A_{n}^{1} \mid \psi^{*}(p)-\langle p,(\bar{a}, \bar{y})\rangle \leqq \bar{\mu}\right\}=W_{0} \cap\left\{p \in A_{n}^{1} \mid \Phi_{m, M}(p) \leqq\langle p,(\bar{a}, \bar{y})\rangle+\bar{\mu}\right\}
$$

is bounded. Let

$$
W_{1}=\left\{p \in A_{n}^{1} \mid\langle p,(\bar{a}, \bar{y})\rangle+\bar{\mu} \geqq \mu\right\},
$$

where $\bar{\mu}\rangle \mu-\langle\bar{p},(\bar{a}, \bar{y})\rangle$. Then $W_{1}$ is a weak neighborhood of $\bar{p}$ such that

$$
W_{1} \cap C \subset\left\{p \in A_{n}^{1} \mid \Phi_{m, M}(p) \leqq\langle p,(\bar{a}, \bar{y})\rangle+\bar{\mu}\right\} .
$$

It follows that $W=W_{0} \cap W_{1}$ is a weak neighborhood of $\bar{p}$ such that $W \cap C$ is bounded.

4. Support functions and attainable sets. Corollaries 5 and 6 of Theorem 2 reduce the proof of Theorem 1 to the analysis of the conditions $(0,0) \in \mathrm{ri}\left(\operatorname{dom} \varphi_{l, L}\right)$ and $(0,0) \in$ int $\left(\operatorname{dom} \varphi_{l, L}\right)$. These conditions can be related to the conditions in Theorem 1 on the convex sets $C_{l}$ and $C_{L}$ through a study of supporting hyperplanes to $\operatorname{dom} \varphi_{l, L}$.

For a start, we derive a formula in terms of $m$ and $M$ for the support function of $\operatorname{dom} \varphi_{l, L}$ on $A_{n}^{1}$, that is, the function which for each arc $p \in A_{n}^{1}$ gives the supremum over dom $\varphi_{l, L}$ of the linear functional on $A_{n}^{1 *}$ corresponding to $p$. This formula relates the support function of dom $\varphi_{l, L}$ to the support functions of the finitedimensional convex sets $C_{l}=\operatorname{dom} l$ and $D_{L}(t)=\operatorname{dom} L_{t}$. It also provides information on the relationship between the weakly attainable set $C_{L}$ and the attainable set $\operatorname{dom} F_{L}$ (see Corollary 4 of Theorem 3).

A useful fact in our analysis is the result that the support function of the effective domain of a convex function is the recession function of the conjugate convex function (provided that the conjugate is not identically infinite); see [11] and $[6, \S 8$ and $\S 13]$. If $\psi$ is a lower semicontinuous convex function (not identically $+\infty$ ) from a locally convex space $E$ to $R^{1} \cup\{+\infty\}$, the recession function of $\psi$, which 
we denote here by $\hat{\psi}$, is given by the formula

$$
\hat{\psi}(w)=\lim _{\lambda \rightarrow+\infty}\left[\psi\left(w^{\prime}+\lambda w\right)-\psi\left(w^{\prime}\right)\right] / \lambda, \quad w^{\prime} \in \operatorname{dom} \psi .
$$

(The limit is the same no matter which $w^{\prime}$ is chosen in dom $\psi$.) It is known that $\hat{\psi}$ is again a lower semicontinuous, convex function from $E$ to $R^{1} \cup\{+\infty\}$, and that

$$
\hat{\psi}(\lambda w)=\lambda \hat{\psi}(w), \quad \lambda \geqq 0 .
$$

In particular, we shall use the fact that the recession functions $\hat{l}$ and $\hat{m}$ satisfy (in view of the modified conjugacy relation (1.7))

$$
\begin{aligned}
& \hat{m}\left(d_{0}, d_{T}\right)=\sup \left\{\left\langle c_{0}, d_{0}\right\rangle-\left\langle c_{T}, d_{T}\right\rangle \mid\left(c_{0}, c_{T}\right) \in C_{l}\right\}, \\
& \hat{l}\left(c_{0}, c_{T}\right)=\sup \left\{\left\langle c_{0}, d_{0}\right\rangle-\left\langle c_{T}, d_{T}\right\rangle \mid\left(d_{0}, d_{T}\right) \in C_{m}\right\},
\end{aligned}
$$

while the recession functions $\hat{L}(t, \cdot, \cdot)$ and $\hat{M}(t, \cdot, \cdot)$ of $L(t, \cdot, \cdot)$ and $M(t, \cdot, \cdot)$ satisfy (in view of (1.8))

$$
\begin{aligned}
& \hat{M}(t, p, s)=\sup \left\{\langle x, s\rangle+\langle v, p\rangle \mid(x, v) \in D_{L}(t)\right\}, \\
& \hat{L}(t, x, v)=\sup \left\{\langle x, s\rangle+\langle v, p\rangle \mid(p, s) \in D_{M}(t)\right\} .
\end{aligned}
$$

Proposition 6. The recession functions $\hat{l}$ and $\hat{L}$ satisfy conditions (A), (B), (C) and (D), and so do $\hat{m}$ and $\hat{M}$. The Bolza functionals $\Phi_{\hat{l}, \hat{L}}$ and $\Phi_{\hat{m}, \hat{M}}$ are thus well defined on $A_{n}^{1}$, and in fact $\Phi_{i, \hat{L}}$ is the recession function of $\Phi_{l, L}\left(\right.$ if $\left.\Phi_{l, L} \not \equiv+\infty\right)$, and $\Phi_{\hat{m}, \hat{M}}$ is the recession function of $\Phi_{m, M}\left(\right.$ if $\left.\Phi_{m, M} \not \equiv+\infty\right)$. Moreover, $\Phi_{\hat{m}, \hat{M}}$ is the support function of $\operatorname{dom} \varphi_{l, L}$, while $\Phi_{i, \hat{L}}$ is the support function of $\operatorname{dom} \varphi_{m, M}$.

Proof. Clearly $\hat{l}$ and $\hat{L}$ satisfy (A), while (D) is trivially satisfied because $\mathcal{L}(t, 0,0) \equiv 0$. If $p, s$ and $\alpha$ are functions having the property in (C) for $L$, then $p, s$ and 0 have the same property for $\hat{L}$; thus $\hat{L}$ satisfies (C). Since $L$ satisfies the measurability condition (B) and $\hat{L}$ can be expressed by definition (see (4.1)) as a limit of difference quotients of $L, \hat{L}$ likewise satisfies (B) (the limit of a sequence of measurable functions being measurable). If $x$ is an arc such that $\Phi_{l, L}(x)<+\infty$, we have

$$
\begin{aligned}
\lim _{\lambda \rightarrow+\infty}[ & \left.\Phi_{l, L}(x+\lambda z)-\Phi_{l, L}(x)\right] / \lambda \\
= & \lim _{\lambda \rightarrow+\infty}[l(x(0)+\lambda z(0), x(T)+\lambda z(T))-l(x(0), x(T))] / \lambda \\
& +\lim _{\lambda \rightarrow+\infty} \int_{0}^{T}([L(t, x(t)+\lambda z(t), \dot{x}(t)+\lambda \dot{z}(t))-L(t, x(t), \dot{x}(t))] / \lambda) d t .
\end{aligned}
$$

The last limit can be exchanged with the integral, because the difference quotient is nondecreasing in $\lambda$ (due to the convexity of $L(t, \cdot, \cdot)$ ). Therefore

$$
\hat{\Phi}_{l, L}(z)=\hat{l}(z(0), z(T))+\int_{0}^{T} \hat{L}(t, z(t), \dot{z}(t)) d t=\Phi_{\hat{l}, \mathcal{L}}(z)
$$

as claimed. It follows that $\Phi_{\hat{l}, \hat{L}}$ is the support function of the convex set dom $\Phi_{l, L}^{*}$ 
in $A_{n}^{1 *}$ [11, Corollary 3D]. But dom $\Phi_{l, L}^{*}$ and $\operatorname{dom} \varphi_{m, M}$ have the same weak* closure in $A_{n}^{1 *}$, because $\Phi_{l, L}=\varphi_{m, M}^{*}$ and $\Phi_{l, L} \not \equiv+\infty$. Hence $\Phi_{\hat{l}, \hat{L}}$ is also the support function of $\operatorname{dom} \varphi_{m, M}$.

Unfortunately, this argument does not yield the useful fact that $\Phi_{\hat{l}, \hat{L}}$ is the support function of dom $\varphi_{m, M}$ even in the case where $\Phi_{l, L}$ is identically $+\infty$. However, to cover the latter case we can replace $m$ and $M$ by

$$
m^{0}\left(d_{0}, d_{T}\right)=\max \left\{m\left(d_{0}, d_{T}\right), 0\right\}, \quad M^{0}(t, p, s)=\max \{M(t, p, s), 0\},
$$

taking $l^{0}$ and $L^{0}$ to be the functions dual to $m^{0}$ and $M^{0}$. Then (A), (B), (C) and (D) are satisfied. The function $\varphi_{m^{0}, M^{0}}$ is nonnegative, so that its conjugate $\Phi_{l^{0}, L^{0}}$ on $A_{n}^{1}$ is finite at the origin, and the result already proved can be applied. Since $C_{m^{0}}=C_{m}$ and $D_{M^{0}}(t)=D_{M}(t)$, we have $\hat{l}^{0}=\hat{l}$ and $\hat{L}^{0}=\hat{L}$ (see (4.4) and (4.6)). The recession function of $\Phi_{l^{0}, L^{0}}$, which is the support function of dom $\varphi_{m^{0}, M^{0}}$, is therefore $\Phi_{i, \hat{L}}$. But dom $\varphi_{m^{0}, M^{0}}$ is the same as dom $\varphi_{m, M}$, and the proof is now complete.

We call an $\operatorname{arc} z \in A_{n}^{1}$ a lineality arc for $l$ and $L$ if there exist real numbers $\alpha_{0}$ and $\alpha_{1}(t)$ (for almost all $t$ ) such that

$$
\begin{aligned}
& l\left(c_{0}+\lambda z(0), c_{T}+\lambda z(t)\right)=l\left(c_{0}, c_{T}\right)+\lambda \alpha_{0} \quad \text { for all } c_{0}, c_{T}, \lambda, \\
& L(t, x+\lambda z(t), v+\lambda \dot{z}(t))=L(t, x, v)+\lambda \alpha_{1}(t) \text { for all } x, v, \lambda,
\end{aligned}
$$

or equivalently if

$$
\begin{aligned}
\hat{l}(-z(0),-z(T)) & =-\hat{l}(z(0), z(T)), \\
\hat{L}(t,-z(t),-\dot{z}(t)) & =-\hat{L}(t, z(t), \dot{z}(t)), \text { a.e. }
\end{aligned}
$$

It is not difficult to verify (using the convexity and positive homogeneity of $\hat{l}$ and $\hat{L}(t, \cdot,:))$ that $z$ has this property if and only if

$$
\Phi_{i, \hat{L}}(-z)=-\Phi_{i, \hat{L}}(z) .
$$

If $\Phi_{l, L}$ is not identically $+\infty$, so that $\Phi_{\hat{l}, \hat{L}}$ is the recession function of $\Phi_{l, L}$ (Proposition 6), property (4.17) characterizes the lineality arcs for $l$ and $L$ as the $\operatorname{arcs} z \in A_{n}^{1}$ for which there is a constant $\alpha$ such that

$$
\Phi_{l, L}(x+\lambda z)=\Phi_{l, L}(x)+\lambda \alpha \text { for all } x \in A_{n}^{1}, \lambda \in R^{1} .
$$

Lineality arcs for $m$ and $M$ are defined analogously.

Ordinarily one would not expect to encounter nonzero lineality arcs in a wellformulated problem of Bolza, but they might occur in certain derived problems, such as the dual problem. Their theoretical role is explained by the next result.

Proposition 7. Assume that $\left(\mathrm{D}_{0}\right)$ holds. Then a continuous linear functional on $A_{n}^{1 *}$ is constant on dom $\varphi_{l, L}$ if and only if it corresponds to an element of $A_{n}^{1}$ which is a lineality arc for $m$ and $M$. The lineality arcs for $m$ and $M$ thus form a subspace of $A_{n}^{1}$ whose dimension is the ( finite) codimension of aff ( $\left.\operatorname{dom} \varphi_{l, L}\right)$ in $A_{n}^{1}$. 
Moreover, it is possible to find matrices $A(t)$ (not necessarily unique) whose components are summable in $t$, such that every lineality arc $z$ for $m$ and $M$ is a solution to the differential equation

$$
\dot{z}(t)=A(t) z(t), \quad(z(0),-z(T)) \in N_{l}
$$

(but not necessarily conversely). Here $N_{l}$ is the orthogonal complement in $R^{n} \times R^{n}$ of the subspace parallel to aff $C_{l}$.

Proof. Since $\left(D_{0}\right)$ holds, every continuous linear functional which is constant on dom $\varphi_{l, L}$ corresponds by Corollary 2 of Theorem 2 to an element of $A_{n}^{1}$. The first part of the proposition is then apparent from the fact that $\Phi_{\hat{m}, \hat{M}}$ is the support function of dom $\varphi_{l, L}$ (Proposition 6) on $A_{n}^{1}$, together with the characterization of lineality arcs for $m$ and $M$ as the $\operatorname{arcs} z$ with the property that

$$
\Phi_{\hat{m}, \hat{M}}(-z)=-\Phi_{\hat{m}, \hat{M}}(z) .
$$

To prove the second part, we take any nonempty, bounded, open set $X$ in $R^{n}$ and select $B(t)$ and $b(t)$ as in Proposition 3. If $z$ is a lineality arc for $m$ and $M$, then properties (4.15) and (4.16) (with $l$ and $L$ replaced by $m$ and $M$ ) imply via (4.3) and (4.5) that the linear functions

$$
\left(c_{0}, c_{T}\right) \rightarrow\left\langle c_{0}, z(0)\right\rangle+\left\langle c_{T},-z(T)\right\rangle, \quad(x, v) \rightarrow\langle x, \dot{z}(t)\rangle+\langle v, z(t)\rangle,
$$

are constant on $C_{l}$ and $D_{L}(t)$, respectively. Therefore $(z(0),-z(t))$ belongs to $N_{l}$, and the expression

$$
\langle x, \dot{z}(t)\rangle+\langle B(t) x+b(t), z(t)\rangle, \quad x \in X,
$$

is for each $t$ a constant independent of $x$. Since $X$ has a nonempty interior, the latter fact implies that $\dot{z}(t)=A(t) z(t)$ for almost every $t$, where $A(t)$ is the transpose of $B(t)$.

Corollary 1. Assume that $\left(\mathrm{D}_{0}\right)$ holds. The convex set dom $\varphi_{l, L}$ has a nonempty interior (so that int dom $\varphi_{l, L}$ and $\mathrm{ri}$ dom $\varphi_{l, L}$ are the same) if and only if there are no lineality arcs for $m$ and $M$ other than the zero arc.

Proof. This follows from the properties of dom $\varphi_{l, L}$ established in Theorem 2.

Corollary 2. Assume that $\left(\mathrm{D}_{0}\right)$ holds. If $z$ is a lineality arc for $m$ and $M$ such that $z(t)=0$ for some $t \in[0, T]$, then $z$ must be the zero arc.

Proof. This is true because $z$ satisfies the linear differential equation (4.19).

Corollary 3. If $\left(\mathrm{D}_{0}\right)$ holds, the codimension of aff $\operatorname{dom} \varphi_{l, L}$ in $A_{n}^{1 *}$ does not exceed $n$, and it is not greater than the codimension of aff $C_{l}$ in $R^{n} \times R^{n}$.

Proof. The codimension in question cannot exceed the dimension of the space of solutions to (4.19). Of course, the dimension of $N_{l}$ is the codimension of aff $C_{l}$.

We are ready now to prove the main result of this section. 
THEOREM 3. If $\left(\mathrm{D}_{0}\right)$ holds, the following statements are equivalent:

(a) The origin of $A_{n}^{1 *}$ belongs to ri dom $\varphi_{l, L}$.

(b) ri $C_{l} \cap$ ri $C_{L} \neq \varnothing$.

(c) There are no arcs $z \in A_{n}^{1}$, other than lineality arcs for $m$ and $M$, with the property that

$$
\hat{m}(z(0), z(T))+\int_{0}^{T} \hat{M}(t, z(t), \dot{z}(t)) d t \leqq 0,
$$

and for every such arc equality holds in (4.21).

The following statements are also equivalent under $\left(\mathrm{D}_{0}\right)$ :

(a') The origin of $A_{n}^{1 *}$ belongs to int dom $\varphi_{l, L}$.

(b') ri $C_{l} \cap$ ri $C_{L} \neq \varnothing$ and aff $\left(C_{l} \cup C_{L}\right)=R^{n} \times R^{n}$.

(c') There are no arcs $z \in A_{n}^{1}$, other than $z=0$, such that (4.21) holds.

Proof. We demonstrate first that (a) and (c) are equivalent. Since ri dom $\varphi_{l, L}$ is nonempty and aff dom $\varphi_{l, L}$ is closed (Theorem 2), the origin fails to belong to ri dom $\varphi_{l, L}$ if and only if it can be separated properly from dom $\varphi_{l, L}$ by some continuous linear functional on $A_{n}^{1 *}$. Such a linear functional necessarily corresponds to an arc $z$ in $A_{n}^{1}$ by Corollary 2 of Theorem 2, and its supremum over $\operatorname{dom} \varphi_{l, L}$ is therefore $\Phi_{\hat{m}, \hat{M}}(z)$ by Proposition 6. It is constant on dom $\varphi_{l, L}$ if and only if $z$ is a lineality arc for $m$ and $M$ (Proposition 7). Thus (a) holds if and only if every arc $z \in A_{n}^{1}$ satisfying $\Phi_{\hat{m}, \hat{M}}(z) \leqq 0$ is a lineality arc for $m$ and $M$ such that $\Phi_{\hat{m}, \hat{M}}(z)=0$. In other words, (a) holds if and only if (c) holds.

If (a) holds, we have $\varphi_{l, L}(0,0)<+\infty$, so that $\Phi_{l, L}$ is not identically $+\infty$ and there exists at least one arc $x$ satisfying (1.3). Therefore, in proving the equivalence of (b) with (a) and (c), it can be assumed that $C_{L} \neq \varnothing$. Then (b) holds if and only if the convex sets $C_{l}$ and $C_{L}$ cannot be separated properly [6, Theorem 11.3]. In other words, if we define $F$ on $R^{n} \times R^{n}$ by

$$
F\left(d_{0}, d_{T}\right)=\inf \left\{\left\langle c_{0}, d_{0}\right\rangle-\left\langle c_{T}, d_{T}\right\rangle \mid\left(c_{0}, c_{T}\right) \in C_{L}\right\}
$$

and make use of (4.3), we can express (b) equivalently as the condition that

$$
\hat{m}\left(d_{0}, d_{T}\right)-F\left(d_{0}, d_{T}\right) \leqq 0 \text { implies } \hat{m}\left(-d_{0},-d_{T}\right)-F\left(-d_{0},-d_{T}\right) \leqq 0 .
$$

To prove that (4.23) is equivalent to (c), it suffices to demonstrate that

$$
-F\left(d_{0}, d_{T}\right)=\min \left\{\int_{0}^{T} \hat{M}(t, z(t), \dot{z}(t)) d t \mid z \in A_{n}^{1}, z(0)=d_{0}, z(T)=d_{T}\right\}
$$

Fix any $\left(d_{0}, d_{T}\right) \in R^{n} \times R^{n}$, and define

$$
\begin{aligned}
l^{0}\left(c_{0}, c_{T}\right) & =\left\langle c_{0}, d_{0}\right\rangle-\left\langle c_{T}, d_{T}\right\rangle, \\
L^{0}(t, x, v) & =0 \quad \text { if }(x, v) \in \operatorname{cl} D_{L}(t), \\
& =+\infty \quad \text { if }(x, v) \notin \operatorname{cl} D_{L}(t) .
\end{aligned}
$$


These functions again satisfy $(A),(B),(C)$ and $\left(D_{0}\right)$, and the functions dual to them are

$$
\begin{gathered}
m^{0}\left(d_{0}^{\prime}, d_{T}^{\prime}\right)=0 \quad \text { if } d_{0}^{\prime}=d_{0} \text { and } d_{T}^{\prime}=d_{T}, \\
=+\infty \quad \text { if } d_{0}^{\prime} \neq d_{0} \text { or } d_{T}^{\prime} \neq d_{T}, \\
M^{0}(t, p, s)=\hat{M}(t, p, s)
\end{gathered}
$$

(the last by (4.5)). In terms of these functions, the relation (4.24) that we want to prove can be written as

$$
-\inf \left\{\Phi_{l^{0}, L^{0}}(x) \mid x \in A_{n}^{1}\right\}=\min \left\{\Phi_{m^{0}, M^{0}}(z) \mid z \in A_{n}^{1}\right\} .
$$

This holds by Corollary 5 of Theorem 2 if

$$
(0,0) \in \text { ri dom } \varphi_{l^{0}}, L^{0} .
$$

Since (a) and (c) have already been shown to be equivalent, we can apply this fact to $l^{0}$ and $L^{0}$ to express (4.29) as condition (c) for $\hat{m}^{0}\left(=\delta_{(0,0)}\right)$ and $\hat{M}^{0}(=\hat{M})$. In this way we reduce the argument to showing that there are no $\operatorname{arcs} z$, other than $z=0$, with the property that

$$
z(0)=0, \quad z(T)=0, \quad \text { and } \quad \int_{0}^{T} \hat{M}(t, z(t), \dot{z}(t)) d t \leqq 0 .
$$

(A lineality arc for $m^{0}$ and $M^{0}$ satisfying (4.30) must be the zero arc by Corollary 2 of Proposition 7.)

Assume that $z \in A_{n}^{1}$ satisfies (4.30), but that $z$ is not the zero arc. We argue from this to a contradiction. Since $C_{L} \neq \varnothing$, there is at least one arc $x^{0} \in A_{n}^{1}$ such that

$$
\left(x^{0}(t), \dot{x}^{0}(t)\right) \in \operatorname{cl} D_{L}(t) \text { a.e. on }[0, T] .
$$

From (4.5) we see that

$$
\begin{aligned}
\hat{M}(t, z(t), \dot{z}(t)) & \geqq\left\langle x^{0}(t), \dot{z}(t)\right\rangle+\left\langle\dot{x}^{0}(t), z(t)\right\rangle \\
& =(d / d t)\left\langle x^{0}(t), z(t)\right\rangle
\end{aligned}
$$

for almost every $t$, where equality holds if and only if

$$
\left\langle x-x^{0}(t), \dot{z}(t)\right\rangle+\left\langle v-\dot{x}^{0}(t), z(t)\right\rangle \leqq 0, \quad \forall(x, v) \in D_{L}(t) .
$$

Subtracting the right side of (4.32) from the left side and integrating over $[0, T]$, we obtain from (4.30) that

$$
0 \leqq \int_{0}^{T} \hat{M}(t, z(t), \dot{z}(t)) d t+\left\langle x^{0}(0), 0\right\rangle-\left\langle x^{0}(T), 0\right\rangle \leqq 0 .
$$

This implies that equality must in fact hold in (4.32) for almost every $t$, so that (4.33) is true for almost every $t$. Since $z$ is not the zero arc, we can find numbers $t_{0}$ and $t_{1}\left(0 \leqq t_{0}<t_{1} \leqq T\right)$ such that $z\left(t_{0}\right)=0$, but $z(t) \neq 0$ for $t \in\left(t_{0}, t_{1}\right]$. For each 
$\tau \in\left(t_{0}, t_{1}\right]$, let $K(\tau)$ denote the set of all vectors $c \in R^{n}$ such that there exists an absolutely continuous arc $x:\left[t_{0}, \tau\right] \rightarrow R^{n}$ with

$$
x(\tau)=c \quad \text { and } \quad(x(t), \dot{x}(t)) \in \operatorname{cl} D_{L}(t) \quad \text { a.e. on }\left[t_{0}, \tau\right] .
$$

Clearly $K(\tau)$ is a convex set containing $x^{0}(\tau)$. If $x$ is any arc satisfying (4.34), the inequality (4.33) implies that

$$
\begin{aligned}
0 & \geqq\left\langle x(t)-x^{0}(t), \dot{z}(t)\right\rangle+\left\langle\dot{x}(t)-\dot{x}^{0}(t), z(t)\right\rangle \\
& =(d / d t)\left\langle x(t)-x^{0}(t), z(t)\right\rangle .
\end{aligned}
$$

Thus $\left\langle x(t)-x^{0}(t), z(t)\right\rangle$ is nonincreasing as a function of $t \in\left[t_{0}, \tau\right]$, so that

$$
\left\langle c-x^{0}(\tau), z(\tau)\right\rangle=\left\langle x(\tau)-x^{0}(\tau), z(\tau)\right\rangle \leqq\left\langle x\left(t_{0}\right)-x^{0}\left(t_{0}\right), z\left(t_{0}\right)\right\rangle=0 .
$$

Therefore $z(\tau)$ is a (nonzero) normal to $K(\tau)$ at $x^{0}(\tau)$ for $t_{0}<\tau \leqq t_{1}$. To contradict this, we show that $x^{0}(\tau)$ is actually an interior point of $K(\tau)$ if $\tau$ is sufficiently near to $t_{0}$. Let $X$ be any bounded, open neighborhood of $x^{0}\left(t_{0}\right)$ in $R^{n}$, and take $B(t)$ and $b(t)$ as in Proposition 3. For each point $a \in R^{n}$, the differential equation

$$
\dot{x}(t)=B(t) x(t)+b(t), \quad x\left(t_{0}\right)=a,
$$

has a unique solution on $[0, T]$, and if $a \in X$ one has $x(t) \in X$ (and hence $(x(t), \dot{x}(t)) \in D_{L}(t)$ by the choice of $B(t)$ and $\left.b(t)\right)$ for $t$ sufficiently near to $t_{0}$. Thus, if the solution to (4.36) is written in the form $x(t)=W_{t}(a)$, we have $W_{\tau}(a) \in K(\tau)$ whenever $a \in X$ and $\tau$ is sufficiently near to $t_{0}\left(\tau>t_{0}\right)$. Let $U$ and $V$ be compact subsets of $X$ such that $x^{0}\left(t_{0}\right) \in$ int $U$ and $U \subset$ int $V$. Then there exists a $\tau, t_{0}<\tau<t_{1}$, such that

$$
x^{0}(t) \in \text { int } U \subset W_{t}(V) \subset X, \quad t_{0} \leqq t \leqq \tau .
$$

These relations imply that

$$
x^{0}(\tau) \in \text { int } W_{\tau}(V) \subset K(\tau),
$$

so that $x^{0}(\tau)$ is an interior point of $K(\tau)$. This completes the proof of the equivalence of (b) with (a) and (c).

The equivalence of $\left(a^{\prime}\right)$ and $\left(c^{\prime}\right)$ is obvious from Corollary 1 of Proposition 7 and the equivalence of (a) and (c). The equivalence of $\left(b^{\prime}\right)$ and $\left(c^{\prime}\right)$ follows, like the equivalence of (b) and (c), from the support function formula (4.24) established above. Theorem 3 is now established.

Corollary 1. Assume that $\left(\mathrm{C}_{0}\right)$ holds. Then one has ri $C_{m} \cap$ ri $C_{M} \neq \varnothing$ if and only if there are no arcs $z \in A_{n}^{1}$, other than lineality arcs for $l$ and $L$, such that

$$
\hat{l}(z(0), z(T))+\int_{0}^{T} \hat{L}(t, z(t), \dot{z}(t)) d t \leqq 0,
$$

and for every such arc equality holds in (4.37).

One has both ri $C_{m} \cap$ ri $C_{M} \neq \varnothing$ and aff $\left(C_{m} \cup C_{M}\right)=R^{n} \times R^{n}$ if and only if there is no arc $z$, other than $z=0$, satisfying (4.37). 
Corollary 2. Assume that $\left(\mathrm{C}_{0}\right)$ holds. Then one has $C_{M} \neq \varnothing$ if and only if there is no arc $z \in A_{n}^{1}$, other than $z=0$, such that

$$
z(0)=0, \quad z(T)=0, \quad \text { and } \int_{0}^{T} \hat{L}(t, z(t), \dot{z}(t)) d t \leqq 0 .
$$

Proof. Invoke the equivalence of $\left(b^{\prime}\right)$ and $\left(c^{\prime}\right)$ for the dual functions in the case where $l$ is the indicator of the origin, so that $m$ is identically 0 .

Corollary 3. Suppose that

$$
L(t, x, v) \geqq L^{1}(t, x, v)+g(t, v-A(t) x),
$$

where $L^{1}$ satisfies (A), (B) and (C), $g$ is a normal convex integrand on $[0, T] \times R^{n}$ whose conjugate $\mathrm{g}^{*}$ has the property that $\mathrm{g}^{*}(t, p)$ is finite and summable in $t$ for every $p \in R^{n}$, and $A(t)$ is an $n \times n$ matrix whose components are summable functions of $t$. Suppose further that the differential equation

$$
\dot{z}(t)=A(t) z(t) \text { for almost every } t \in[0, T]
$$

has no solutions $z$, other than lineality arcs for $l$ and $L^{1}$, such that

$$
\hat{l}(z(0), z(T))+\int_{0}^{T} \hat{L}^{1}(t, z(t), \dot{z}(t)) d t \leqq 0,
$$

and that for every such solution equality holds in (4.41).

Then $\left(\mathrm{C}_{0}\right)$ holds and ri $C_{m} \cap$ ri $C_{M} \neq \varnothing$, so that the hypothesis of Theorem 1(a) is satisfied. If in fact there is no nonzero solution $z$ to (4.40) which is a lineality arc for $l$ and $L^{1}$, then also aff $\left(C_{m} \cup C_{M}\right)=R^{n} \times R^{n}$.

Proof. Since $L^{1}$ satisfies (C), there exist functions $\bar{s} \in L_{n}^{1}, \bar{p} \in L_{n}^{\infty}$ and $\alpha \in L_{1}^{1}$ such that

$$
L(t, x, v) \geqq\langle x, \bar{s}(t)\rangle+\langle v, \bar{p}(t)\rangle-\alpha(t)+g(t, v-A(t) x) .
$$

Let $L^{0}(t, x, v)$ denote the right side of (4.42). The Hamiltonian $H^{0}$ corresponding to $L^{0}$ is

$$
H^{0}(t, x, p)=\alpha(t)-\langle x, \bar{s}(t)\rangle+\langle A(t) x, p-\bar{p}(t)\rangle+g^{*}(t, p-\bar{p}(t)),
$$

and this is summable in $t$ for every $x$ and $p$ (apply [7, II, Corollary 2A] to $g^{*}$, using the hypothesis that $g^{*}(t, p)$ is summable in $t$ for each $\left.p\right)$. Corollary 1 of Proposition 2 implies in this case that $\left(C_{0}\right)$ holds. The inequality (4.39) implies at the same time (from the definition of the recession function of a convex function) that

$$
\hat{L}(t, x, v) \geqq \hat{L}^{1}(t, x, v)+\hat{g}(t, v-A(t) x),
$$

where $\hat{g}(t, \cdot)$ is the recession function of $g(t, \cdot)$. But $g(t, \cdot)$ is cofinite; hence

$$
\begin{aligned}
\hat{g}(t, u) & =0 & & \text { if } u=0, \\
& =+\infty & & \text { if } u \neq 0 .
\end{aligned}
$$


It follows that if $z \in A_{n}^{1}$ is any arc satisfying (4.37), then (4.40) and (4.41) hold, and the conclusions of the corollary are apparent.

REMARK. The assumptions in Corollary 3 concerning the differential equation (4.40) are satisfied if

$$
\hat{l}\left(c_{0}, c_{T}\right)<+\infty \text { implies that } c_{0}=0 \text { or } c_{T}=0,
$$

as is true in particular if $C_{l}$ has a bounded image under either of the projections $\left(c_{0}, c_{T}\right) \rightarrow c_{0}$ or $\left(c_{0}, c_{T}\right) \rightarrow c_{T}$. Indeed, in this case an arc $z$ satisfying (4.40) and (4.41) has either $z(0)=0$ or $z(T)=0$, and consequently $z$ is the zero arc.

Corollary 4. Assume that $\left(\mathrm{C}_{0}\right)$ is satisfied. Then, for the convex function $F_{L}$ in (1.25), the convex set $\operatorname{dom} F_{L}$ (the set of attainable endpoints for $L$ ) is related to $C_{L}$ (the set of weakly attainable endpoints for $L$ ) by

$$
\operatorname{dom} F_{L} \subset C_{L} \subset \operatorname{cldom} F_{L} .
$$

Thus in particular one has

$$
\text { ri } C_{L}=\text { ri dom } F_{L} \text {. }
$$

Proof. The first inclusion in (4.47) is obvious, so we need only show that $\operatorname{dom} F_{L} \supset$ ri $C_{L}$. Let $\left(\bar{c}_{0}, \bar{c}_{T}\right)$ be any point of ri $C_{L}$ and take

$$
\begin{aligned}
l\left(c_{0}, c_{T}\right) & =0 & & \text { if } c_{0}=\bar{c}_{0} \text { and } c_{T}=\bar{c}_{T}, \\
& =+\infty & & \text { if } c_{0} \neq \bar{c}_{0} \text { or } c_{T} \neq \bar{c}_{T} .
\end{aligned}
$$

Then (b) is satisfied in Theorem 3, and it follows that (a) is satisfied as well, so that $\varphi_{l, L}(0,0)<+\infty$. Thus there is at least one arc $x \in A_{n}^{1}$ with $\Phi_{l, L}(x)<+\infty$. In view of the definition of $l$, such an arc has

$$
x(0)=\bar{c}_{0}, \quad x(T)=\bar{c}_{T} \quad \text { and } \quad \int_{0}^{T} L(t, x(t), \dot{x}(t)) d t<+\infty .
$$

Therefore $\left(\bar{c}_{0}, \bar{c}_{T}\right) \in \operatorname{dom} F_{L}$.

Proof of Theoren 1. There is nothing left to do, except to combine Theorem 3 with Corollaries 5 and 6 of Theorem 2.

5. Dual interpretation of optimal arcs. The fact that the arcs which minimize $\Phi_{m, M}$ over $A_{n}^{1}$ correspond to the subgradients (in $A_{n}^{1}$ ) of $\varphi_{l, L}$ at the origin of $A_{n}^{1 *}$ has already been noted in Proposition 5 in $\S 3$. Dually, the arcs which minimize $\Phi_{l, L}$ correspond to subgradients of $\varphi_{m, M}$ at the origin. These facts, in conjunction with Theorem 2, make it possible to interpret the optimal arcs in a given problem of Bolza in terms of the differential effect of perturbations on the infimum in the dual problem of Bolza, and vice versa. Analogous duality results have been developed by the author in the general theory of convex programs $[6, \S 30]$.

We denote by $\varphi_{l, L}^{\prime}(\bar{a}, \bar{y} ; a, y)$ the one-sided directional derivative of $\varphi_{l, L}$ with respect to $(a, y)$ at $(\bar{a}, \bar{y})$ :

$$
\varphi_{l, L}^{\prime}(\bar{a}, \bar{y} ; a, y)=\lim _{\lambda \downarrow 0}\left[\varphi_{l, L}(\bar{a}+\lambda a, \bar{y}+\lambda y)-\varphi_{l, L}(\bar{a}, \bar{y})\right] / \lambda .
$$


Since $\varphi_{l, L}$ is convex, this is well defined, provided that $\varphi_{l, L}(\bar{a}, \bar{y})$ is finite. An arc $p \in A_{n}^{1}$ belongs to the subgradient set $\partial \varphi_{l, L}(\bar{a}, \bar{y})$ if and only if

$$
\begin{aligned}
\varphi_{l, L}^{\prime}(\bar{a}, \bar{y} ; a, y) \geqq\langle(a, y), p\rangle=\langle a, p(0)\rangle+\int_{0}^{T}\langle y(t), \dot{p}(t)\rangle d t & \text { for all }(a, y) \in A_{n}^{1 *} .
\end{aligned}
$$

The following result is stated one-sidedly, but needless to say the dual assertions are also valid, where $l$ and $L$ are interchanged with $m$ and $M$, and $\left(\mathrm{D}_{0}\right)$ is replaced by $\left(C_{0}\right)$. These dual assertions characterize the case where there is a unique optimal arc for $l$ and $L$.

By a minimizing sequence for $\Phi_{m, M}$, we mean of course a sequence of arcs $p_{k}$ in $A_{n}^{1}$ such that $\Phi_{m, M}\left(p_{k}\right)$ tends to the infimum of $\Phi_{m, M}$ over $A_{n}^{1}$. If the level sets of $\Phi_{m, M}$ are weakly compact as in Theorem 1(b), such a sequence has cluster points, and every cluster point is an $\operatorname{arc} p$ which minimizes $\Phi_{m, M}$.

TheOREM 4. Assume that $\left(\mathrm{D}_{0}\right)$ holds, and let $P$ denote the (closed, convex) subset of $A_{n}^{1}$ consisting of the arcs $p$ which minimize $\Phi_{m, M}$.

(a) If one of the equivalent conditions (a), (b) or (c) in Theorem 3 is satisfied and $\varphi_{l, L}(0,0)$ (the infimum of $\left.\Phi_{l, L}\right)$ is finite, then for every $a \in R^{n}$ and $y \in L_{n}^{\infty}$, one has

$$
\varphi_{l, L}^{\prime}(0,0 ; a, y)=\sup \{\langle(a, y), p\rangle \mid p \in P\} .
$$

(Thus $\varphi_{l, L}^{\prime}(0,0 ; \cdot, \cdot)$ is the support function of $P$.)

(b) $P$ consists of a single element $p$ (that is, $p$ is the unique optimal arc for $m$ and $M$ ) if and only if $\varphi_{l, L}$ is (finite and) differentiable at $(0,0)$ in the sense of Gateaux. In this event $p$ is the gradient of $\varphi_{l, L}$ at $(0,0)$ in the sense of Gâteaux: for every $a \in R^{n}$ and $y \in L_{n}^{\infty}$ one has

$$
\varphi_{l, L}^{\prime}(0,0 ; a, y)=\langle(a, y), p\rangle=\langle a, p(0)\rangle+\int_{0}^{T}\langle y(t), \dot{p}(t)\rangle d t .
$$

(c) In the situation in (b), every minimizing sequence for $\Phi_{m, M}$ converges to $p$ in the weak topology of $A_{n}^{1}$. In order that $p$ actually be the gradient in the sense of Fréchet, it is necessary and sufficient that every minimizing sequence for $\Phi_{m, M}$ converge to $p$ in the strong topology.

Proof. (a) Theorem 1 and Proposition 5 imply that $P$ consists of the subgradients of $\varphi_{l, L}$ at $(0,0)$ which belong to $A_{n}^{1}$. On the other hand, Corollary 1 of Theorem 2 asserts that $\varphi_{l, L}$ has no subgradients in $A_{n}^{1 * *} \backslash A_{n}^{1}$. Therefore $P=\partial \varphi_{l, L}(0,0)$. However, since $\varphi_{l, L}$ is continuous at $(0,0)$ relative to aff dom $\varphi_{l, L}$, which is closed (Theorem 2), $\varphi_{l, L}^{\prime}(0,0 ; \cdot, \cdot)$ is the support function of $\partial \varphi_{l, L}(0,0)$. (This fact has been stated by Moreau [3], [4] in the case of a function continuous relative to the whole space, but the extension to the present case is elementary.) Formula (5.3) is thereby proved.

If $P$ consists of a single arc $p$, then condition (c) of Theorem 3 is satisfied, and the minimum of $\Phi_{m, M}$ is finite. Theorem $1(\mathrm{~b})$ then implies that $\varphi_{l, L}(0,0)$ is finite, 
and, applying (a), we see that (5.4) holds. Conversely, if $\varphi_{l, L}(0,0)$ is finite and (5.4) holds, then for every $a \in R^{n}$ and $y \in L_{n}^{\infty}$ it must be true that $\varphi_{l, L}(\lambda a, \lambda y)<+\infty$ for $\lambda$ sufficiently small. Thus aff $\operatorname{dom} \varphi_{l, L}$ is all of $A_{n}^{1 *}$, and we may conclude that $(0,0)$ is an interior point of $\operatorname{dom} \varphi_{l, L}$. The assumptions in (a) are then met, and (5.3) shows that $p$ is the unique element of $P$.

(c) Since one has $(0,0) \in$ int dom $\varphi_{l, L}$ in the situation in (b), $\varphi_{l, L}$ agrees with the conjugate function $\Phi_{m, M}^{*}$ in a neighborhood of $(0,0)$, and neither function takes on $-\infty$ (Corollary 4 to Theorem 2). The assertions are then immediate from the general results relating the rotundity properties of convex functions to the differential properties of their conjugates [1, Theorem 1 and corollaries]. This completes the proof of Theorem 4 .

Theorem 4 explains the exact significance of the optimal arcs in the problem of Bolza dual to a given problem (satisfying the stated conditions). Thus in the simplest case, where the dual problem has a unique optimal arc $p$ and (5.4) holds, the initial point $p(0)$ gives the directional derivatives of the infimum of $\Phi_{l, L}$ with respect to displacements of the form

$$
l(x(0), x(T)) \rightarrow l(x(0)+a, x(T)),
$$

while the derivatives $\dot{p}(t)$ give the directional derivatives of the infimum of $\Phi_{l, L}$ with respect to displacements of the form

$$
L(t, x(t), \dot{x}(t)) \rightarrow L(t, x(t)+y(t), \dot{x}(t)) .
$$

To illustrate further, consider the case where $\left(\mathrm{D}_{0}\right)$ holds, ri $C_{l} \cap$ ri $C_{L} \neq \varnothing$, and an optimal arc $x$ for $l$ and $L$ is known to exist. Corollary 1 of Theorem 1 implies the existence of arcs $p$ such that $x$ and $p$ satisfy the generalized Hamiltonian equation

$$
(-\dot{p}(t), \dot{x}(t)) \in \partial H(t, x(t), p(t)) \quad \text { a.e. }
$$

(see $[9, \S 9]$ and [10]). These arcs $p$ are in fact the optimal arcs for the dual problem. The initial points $p(0)$ that may be chosen in (5.7) are thus characterized by Theorem 4 as the subgradients of the convex function

$$
\varphi(a)=\varphi_{l, L}(a, 0), \quad a \in R^{n},
$$

at $a=0$.

6. Minimization over $A_{n}^{r}$. The preceding results treat minimization over $A_{n}^{1}$, but some conclusions may also be drawn concerning minimization over the spaces $A_{n}^{r}$, where $A_{n}^{r}$ consists of the absolutely continuous arcs $x$ over $[0, T]$ such that $\dot{x}$ belongs to $L_{n}^{r}(1 \leqq r \leqq+\infty)$.

For each $(a, y) \in A_{n}^{1 *}$, let $\varphi_{l, L}^{r}(a, y)$ denote the infimum of the expression (3.3) over all arcs $x \in A_{n}^{r}$. Then $\varphi_{l, L}^{r}$ is a convex function on $A_{n}^{1 *}, \varphi_{l, L}^{r} \geqq \varphi_{l, L}$, and by definition one has

$$
\varphi_{l, L}^{r}(0,0)=\inf _{x \in A_{n}^{r}} \Phi_{l, L}(x) .
$$


We shall make use of the following stronger condition than $\left(D_{0}\right)$ :

$\left(\mathrm{D}_{0}^{r}\right)$ For each $x \in R^{n}$ there exist functions $v \in L_{n}^{r}$ and $\beta \in L_{1}^{1}$ such that $L(t, x, v(t)) \leqq \beta(t)$.

Proposition 8. If $\left(\mathrm{D}_{0}^{r}\right)$ holds, then $\varphi_{l, L}^{r}$ agrees with $\varphi_{l, L}$ everywhere on $A_{n}^{1 *}$, except perhaps at boundary points of $\operatorname{dom} \varphi_{l, L}$ relative to aff dom $\varphi_{l, L}$.

Proof. Since $\varphi_{l, L}^{r}$ majorizes $\varphi_{l, L}$, the convex set epi $\varphi_{l, L}^{r}$ is contained in epi $\varphi_{l, L}$. We shall demonstrate that

$$
\text { cl epi } \varphi_{l, L}^{r} \supset \text { epi } \varphi_{l, L},
$$

that aff epi $\varphi_{l, L}^{r}$ is closed, and that ri epi $\varphi_{l, L}^{r}$ is not empty. This will suffice to establish the proposition, because of the convexity of $\varphi_{l, L}^{r}$ and $\varphi_{l, L}$.

Suppose that $(a, y) \in A_{n}^{1 *}$ and $\mu \in R^{1}$ are such that

$$
\varphi_{l, L}(a, y)<\mu .
$$

To prove (6.2) we construct a sequence of elements $\left(a_{k}, y_{k}\right)$ converging in $A_{n}^{1 *}$ to $(a, y)$ and having the property that

$$
\limsup _{k \rightarrow \infty} \varphi_{l, L}^{r}\left(a_{k}, y_{k}\right)<\mu .
$$

Since (6.3) holds, there exists an $\operatorname{arc} x \in A_{n}^{1}$ such that

$$
l(x(0)+a, x(T))+\int_{0}^{T} L(t, x(t)+y(t), \dot{x}(t)) d t<\mu .
$$

We observe next that Proposition 3 remains valid (by the same argument) if $\left(D_{0}\right)$ is replaced by $\left(\mathrm{D}_{0}^{r}\right)$ and the components of $B(t)$ and $b(t)$ are asserted to be $L^{r}$-summable. Taking $X$ to be a bounded subset of $R^{n}$ containing $x(t)+y(t)$ for almost every $t$, we apply this generalization of Proposition 3 and for the corresponding $B$ and $b$ set

$$
v(t)=B(t)[x(t)+y(t)]+b(t) .
$$

Then $v \in L_{n}^{r}$, and $L(t, x(t)+y(t), v(t))$ is summable in $t$. For each positive integer $k$ let $v_{k}$ be the function in $L_{n}^{r}$ defined by

$$
\begin{aligned}
v_{k}(t) & =\dot{x}(t) & & \text { if }|\dot{x}(t)| \leqq k, \\
& =v(t) & & \text { if }|\dot{x}(t)|>k .
\end{aligned}
$$

Then $L\left(t, x(t)+y(t), v_{k}(t)\right)$ is summable in $t$, and

$$
\lim _{k \rightarrow \infty} \int_{0}^{T} L\left(t, x(t)+y(t), v_{k}(t)\right) d t=\int_{0}^{T} L(t, x(t)+y(t), \dot{x}(t)) d t .
$$

Now define $a_{k} \in R^{n}, y_{k} \in L_{n}^{\infty}$ and $x_{k} \in A_{n}^{r}$ by

$$
x_{k}(T)=x(T), \quad \dot{x}_{k}=v_{k}, \quad a_{k}=x(0)+a-x_{k}(0), \quad y_{k}=x+y-x_{k} .
$$


Then $\left(a_{k}, y_{k}\right)$ converges to $(a, y)$ in $A_{n}^{1 *}$. We have

$$
\varphi_{l, L}^{r}\left(a_{k}, y_{k}\right) \leqq l\left(x_{k}(0)+a_{k}, x_{k}(T)\right)+\int_{0}^{T} L\left(t, x_{k}(t)+y_{k}(t), \dot{x}_{k}(t)\right) d t,
$$

and from (6.5), (6.6), and (6.7) we conclude that (6.4) holds as desired.

The proof that aff dom $\varphi_{l, L}^{r}$ is closed, and ri dom $\varphi_{l, L}^{r}$ is nonempty, is a copy of the proof of the corresponding facts for $\varphi_{l, L}$ in Theorem 2 . The only real difference is that, instead of invoking Proposition 3, one uses the generalization described above.

COROLlaRY. If condition $\left(\mathrm{D}_{0}^{r}\right)$ holds, then Theorem 2 and all its corollaries are valid with $\varphi_{l, L}$ replaced by $\varphi_{l, L}^{r}$ (that is, with $\Phi_{l, L}$ and its perturbations minimized over $A_{n}^{r}$, rather than $A_{n}^{1}$ ).

THEOREM 5. Let $1 \leqq r \leqq+\infty$.

(a) If $\left(\mathrm{D}_{0}^{r}\right)$ holds and ri $C_{l} \cap$ ri $C_{L} \neq \varnothing$, then

$$
\inf _{x \in A_{n}^{1}} \Phi_{l, L}(x)=\inf _{x \in A_{n}^{r}} \Phi_{l, L}(x)=-\min _{p \in A_{n}^{1}} \Phi_{m, M}(p) .
$$

(b) Suppose that the conditions in Corollary 3 of Theorem 3 are satisfied, with the components of $A$ belonging to $L_{n}^{r}$, and with $g$ of the form

$$
g(t, u)=\eta(|u-c(t)|),
$$

where $c \in L_{1}^{r}$, and $\eta$ is a nondecreasing function from $[0,+\infty)$ to $(-\infty,+\infty]$ such that

$$
\liminf _{\lambda \rightarrow+\infty} \eta(\lambda) / \lambda^{r}>0 .
$$

(If $r=+\infty,(6.11)$ is to be replaced by the condition that $\eta(\lambda)=+\infty$ for $\lambda$ sufficiently large.) Then every feasible arc $x$ for $l$ and $L$ belongs to $A_{n}^{r}$, and one has

$$
\min _{x \in A_{n}^{r}} \Phi_{l, L}(x)=\min _{x \in A_{n}^{1}} \Phi_{l, L}(x)=-\inf _{p \in A_{n}^{1}} \Phi_{m, M}(p)=-\inf _{p \in A_{n}^{r}} \Phi_{m, M}(p) .
$$

Proof. Statement (a) is obtained by combining Proposition 8 with Theorem 3 and Corollary 5 of Theorem 2. The assumptions in (b) imply by Corollary 3 of Theorem 3 that the middle equality holds in (6.12), and they also imply (since $L^{1}$ satisfies (C)) the existence of functions $\bar{s} \in L_{n}^{1}, \bar{p} \in L_{n}^{\infty}$ and $\alpha \in L_{1}^{1}$, and constants $\rho>0$ and $\beta$, such that

$$
L(t, x, v) \geqq\langle x, \bar{s}(t)\rangle+\langle v, \bar{p}(t)\rangle-\alpha(t)+\eta_{r}(|v-A(t) x-c(t)| / \rho)-\beta,
$$

where

$$
\begin{aligned}
\eta_{r}(\lambda) & =(1 / r) \lambda^{r} & & \text { for } 1 \leqq r<+\infty \\
\eta_{\infty}(\lambda) & =0 & & \text { if } 0 \leqq \lambda \leqq 1 \\
& =+\infty & & \text { if } 1<\lambda<+\infty
\end{aligned}
$$


If $x$ is an arc in $A_{n}^{1}$ such that $\Phi_{l, L}(x)<+\infty$, it follows from (6.13) that

$$
\eta_{r}(|\dot{x}(t)-A(t) x(t)-c(t)| / \rho) \leqq \mu(t),
$$

where $\mu \in L_{1}^{1}$. Thus

$$
\dot{x}(t)=A(t) x(t)+c(t)+u(t),
$$

where $u \in L_{n}^{r}$. Since the components of $A$ and $c$ are elements of $L_{1}^{r}$, we conclude from (6.15) that $x \in A_{n}^{r}$. This proves the assertion in the theorem concerning $\Phi_{l, L}$, and at the same time it establishes the first equality in (6.12).

Taking conjugates on both sides of (6.13), we see that

$$
M(t, p, s) \leqq \alpha(t)+\langle c(t), p-\bar{p}(t)\rangle+\eta_{q}(\rho|p-\bar{p}(t)|)+\beta
$$

whenever

$$
s-\bar{s}(t)+A^{*}(t)[p-\bar{p}(t)]=0,
$$

where $A^{*}(t)$ is the transpose of $A(t)$ and $(1 / r)+(1 / q)=1$. Define

$$
L^{0}(t, x, v)=L(t, x, v)-\langle x, \bar{s}(t)\rangle-\langle v, \bar{p}(t)\rangle,
$$

so that

$$
M^{0}(t, p, s)=M(t, p+\bar{p}(t), s+\bar{s}(t)) .
$$

Obviously $l$ and $L^{0}$ again satisfy (A), (B), (C), and (D); therefore $m$ and $M^{0}$ again satisfy (A), (B), (C), and (D), and we can apply Proposition 8 to $\varphi_{m, M^{0}}$ and $\varphi_{m, M^{0}}^{r}$. The hypothesis of Proposition 8 is satisfied for these functions, because

$$
M^{0}\left(t, p,-A^{*}(t) p\right) \leqq \alpha(t)+\langle c(t), p\rangle+\eta_{q}(\rho|p|)+\beta
$$

for every $p \in R^{n}$ and $t \in[0, T]$ by (6.16) and (6.17). Thus $\varphi_{m, M^{0}}^{r}$ agrees with $\varphi_{m, M^{0}}$ except at boundary points of $\operatorname{dom} \varphi_{m, M^{0}}$ relative to aff dom $\varphi_{m, M^{0}}$. However, according to (6.19) we have

$$
\varphi_{m, M^{\mathrm{o}}}(a, y)=\varphi_{m, M}(a+\bar{a}, y+\bar{y}), \quad \varphi_{m, M^{\mathrm{o}}}^{r}(a, y)=\varphi_{m, M}^{r}(a+\bar{a}, y+\bar{y}),
$$

where

$$
\bar{y}(t)=\bar{p}(t)+\int_{t}^{T} \bar{s}(\tau) d \tau \text { and } \bar{a}=\int_{0}^{T} \bar{s}(t) d t .
$$

Hence $\varphi_{m, M}^{r}$ agrees with $\varphi_{m, M}$ except at boundary points of dom $\varphi_{m, M}$ relative to aff dom $\varphi_{m, M}$. In particular, since $(0,0) \in \operatorname{ridom} \varphi_{m, M}$ by Theorem 3 and its Corollary 3 , we have $\varphi_{m, M}^{r}(0,0)=\varphi_{m, M}(0,0)$, or in other words the third equality in (6.12).

\section{REFERENCES}

1. E. Asplund and R. T. Rockafellar, Gradients of convex functions, Trans. Amer. Math. Soc. 139 (1969), 443-467. MR 39 \#1968.

2. L. Cesari, Existence theorems for weak and usual optimal solutions in Lagrange problems with unilateral constraints. I, Trans. Amer. Math. Soc. 124 (1966), 369-412. MR 34 \#3392. 
3. J. J. Moreau, Fonctionelles convexes, mimeographed lecture notes, Collège de France, 1967.

4. - Sur la fonction polaire d'une fonction semi-continue supérieurement, C. R. Acad. Sci. Paris 258 (1964), 1128-1130. MR 28 \#3307.

5. C. Olech, Existence theorems for optimal problems with vector-valued cost function, Trans. Amer. Math. Soc. 136 (1969), 159-180. MR 38 \#2655.

6. R. T. Rockafellar, Convex analysis, Princeton Univ. Press, Princeton, N. J., 1969.

7. - Integrals which are convex functionals. I, Pacific J. Math. 24 (1968), 525-540; II, Pacific J. Math. (to appear).

8. - Measurable dependence of convex sets and functions on parameters, J. Math. Anal. Appl. 28 (1969), 4-25. MR 40 \#288.

9. - Conjugate convex functions in optimal control and the calculus of variations, J. Math. Anal. Appl. 32 (1970), 174-222.

10. - Generalized Hamiltonian equations in convex problems of Lagrange, Pacific J. Math. 33 (1970), 411-427.

11. - Level sets and continuity of conjugate convex functions, Trans. Amer. Math. Soc. 123 (1966), 46-63. MR 33 \#544.

12. — Convex Bolza functionals in control problems with state constraints (to appear).

13. J. L. Joly, Une famille de topologies et de convergences sur l'ensemble des fonctionelles convexes, Thèse, Grenoble, 1970.

UNIVERSITY OF WASHINGTON,

SeATtLe, Washington 98105 